\title{
Linear Classifiers Under Infinite Imbalance
}

\author{
Paul Glasserman and Mike Li \\ Columbia Business School
}

June 2021

\begin{abstract}
We study the behavior of linear discriminant functions for binary classification in the infinite-imbalance limit, where the sample size of one class grows without bound while the sample size of the other remains fixed. The coefficients of the classifier minimize an expected loss specified through a weight function. We show that for a broad class of weight functions, the intercept diverges but the rest of the coefficient vector has a finite limit under infinite imbalance, extending prior work on logistic regression. The limit depends on the left tail of the weight function, for which we distinguish three cases: bounded, asymptotically polynomial, and asymptotically exponential. The limiting coefficient vectors reflect robustness or conservatism properties in the sense that they optimize against certain worst-case alternatives. In the bounded and polynomial cases, the limit is equivalent to an implicit choice of upsampling distribution for the minority class. We apply these ideas in a credit risk setting, with particular emphasis on performance in the high-sensitivity and high-specificity regions.
\end{abstract}

\section{Introduction}

Binary classification tasks often face a severe problem of imbalanced data: observations from one class are plentiful but observations from the other class are scarce. In detecting rare diseases, for example, one may have access to nearly unlimited measurements from healthy patients but only a few from sick patients; lenders often have rich data on low-risk borrowers but fewer observations of borrowers who default.

Under extreme imbalance, a simple rule that predicts that all observations are from the majority class achieves near-perfect accuracy on training data. Such a rule is clearly useless in detecting observations from the minority class, which is often the main objective of the classification. In avoiding this trap, we would like to separate the effect of the threshold used for classification from the effect of the features used for scoring observations. We can then correct for imbalance by changing the threshold if we have an effective scoring rule.

In the case of logistic regression, this separation is provided by the intercept and the rest of the coefficient vector. Owen [17] analyzed the behavior of logistic regression in the infinitely imbalanced limit, where the size of the majority class becomes infinite while the size of the 
minority class remains fixed. He showed that the intercept tends to negative infinity, as a consequence of the growing imbalance, but the rest of the coefficient vector approaches a finite limit.

We extend Owen's [17] result to a wide class of linear discriminant functions exhibiting a variety of behaviors. These classifiers assign a score to each observation using a linear combination of features, with coefficients chosen to minimize a loss function; the score is compared to a threshold to classify the observation. In the framework of Eguchi and Copas [5], the loss is determined by a weight function that penalizes high scores for one class and low scores for the other class. We study three broad families of such classifiers, grouped according to the lefttail behavior of the weight function: bounded, asymptotically polynomial, and asymptotically exponential.

Perhaps surprisingly, the asymptotically polynomial and bounded cases (which include logistic regression) all have the same limit — these methods all become equivalent under infinite imbalance. With asymptotically exponential weight functions we get different limits. The exponential case includes, in the case of symmetric penalties, the loss function in the AdaBoost method (as formulated in Freund and Schapire [9], and Friedman, Hastie, and Tibshirani [7]). We will see that asymmetric exponential penalties put more weight on specificity (the true negative rate) or sensitivity (the true positive rate) in the classification task. This flexibility makes the exponential case convenient and easier to tailor to a desired objective.

We also study the form of the limiting coefficient vectors to understand what the infiniteimbalance limit says about the classification rules. We show that the limits reflect robustness properties, in the sense that they are optimized against certain worst-case alternatives. Different types of robustness properties can also be seen as different types of conservatism in selecting which errors to emphasize under extreme imbalance.

These points are easiest to appreciate in the bounded and asymptotically polynomially setting, which includes logistic regression. We know from the Neyman-Pearson lemma that the optimal rule for classifying an observation as coming from one probability distribution or another uses the likelihood ratio between the two distributions. The limiting coefficient vector with a bounded or asymptotically polynomially weight function is the log likelihood ratio between the distribution of the majority class and a "worst-case" alternative. Among the set of distributions having the mean of the minority class, this alternative is the distribution closest to that of the majority class, with closeness measured through relative entropy or Kullback-Leibler divergence. This is the worst case because distributions that are closer are harder to separate. Thus, we show that the limiting coefficient vector provides the best (Neyman-Pearson) classifier for the worst alternative to the majority distribution among all distributions with the mean of the 
minority class.

We also prove a version of this result for the case of asymptotically exponential weight functions. The bounded and asymptotically polynomial cases implicitly emphasize conservatism with respect to false positives in identifying draws from the minority class. The asymptotically exponential case balances concerns about false negatives and false positives, with the relative weight determined by the choice of exponent.

Imbalance is often addressed through downsampling (discarding observations from the majority class) or upsampling (reproducing or creating synthetic observations from the minority class); see, for example, the methods in Chawla et al. [2], Drummond and Holte [4], and Kubat and Matwin [11], and the comparison of methods in Liu, Wu, and Zhou [15]. The infinite imbalance limits we study can also be understood from this perspective in the bounded and asymptotically polynomial cases. Linear discriminant rules for these cases (including logistic regression) become equivalent, in the infinite-imbalance limit, to an implicit choice of upsampling distribution. This implicit rule upsamples the minority class using the worst-case alternative to the majority class.

We illustrate these ideas through numerical examples and an empirical application. As is customary, we examine classification performance through the receiver operating characteristic (ROC) curve. We use partial area-under-the-curve (pAUC) measures, as introduced in McClish [16], to focus attention on the high-specificity and high-sensitivity endpoints. We argue that these regions are where the choice of weights for the discriminant function matters most. Using exponential weight functions, we find a consistent ranking of performance according to the size of the exponent, but the ordering flips between the high-sensitivity and high-specificity regions. Consistent with our limiting results, the behavior of logistic regression becomes similar to that of a classifier from an exponential weight function with small exponent, as the degree of imbalance grows.

We apply these ideas in a credit risk setting using mortgage data from Freddie Mac. We consider the problem of predicting default in the first two years of a loan, using features available at the time the loan was made. Defaults are rare, making the data highly imbalanced. We take the view that in an initial screening, a lender would want to achieve a high level of sensitivity in detecting likely defaulters. We calibrate logistic and exponential classifiers to achieve high true positive rates in training data and then compare true positive and true negative rates in test data. The relative performance of the exponential classifiers and logistic regression are consistent with the predictions from our theoretical analysis.

Section 2 discusses the class of linear discriminant functions we study based on minimizing expected loss measures. Section 3 establishes the existence of unique minimizers for empirical 
loss measures. Section 4 presents our main theoretical results, the coefficient limits under infinite imbalance. Section 5 discusses the interpretation of the limits. Section 6 presents numerical results, and Section 7 develops the credit risk application. Most proofs appear in appendices.

\section{Discriminant Functions}

\section{$2.1 \quad$ Logistically Consistent Objectives}

We consider data in which each observation takes the form of a pair $(x, y) \in \mathbb{R}^{d} \times\{0,1\}$, where $x$ is a vector of features or attributes, and $y$ is a binary class label. When we introduce imbalance, 0 will label the majority class, and 1 will label the minority class. A discriminant function $\eta(x)$ assigns a score to each feature vector $x$, with the intention that points from class 1 will tend to have higher scores than points from class 0 , so that the score can be used for classifying unlabeled observations: an observation $x$ is predicted to be from class 1 if and only if $\eta(x)>t$, for some threshold $t$. A linear discriminant function takes the form $\eta(x)=\alpha+\beta^{\top} x$, for some $\alpha \in \mathbb{R}$ and $\beta \in \mathbb{R}^{d}$. We are primarily interested in the vector $\beta$; if our rule for predicting class 1 based on features $x$ is $\alpha+\beta^{\top} x>t$, then the effect of $\alpha$ can be absorbed into the threshold $t$.

We select $(\alpha, \beta)$ by minimizing a loss function. To formulate the objective, it is useful to introduce distributions $F_{0}$ and $F_{1}$ on $\mathbb{R}^{d}$, describing the distributions of features in the two classes, and marginal probabilities $\pi_{0}$ and $\pi_{1}=1-\pi_{0}$ for the two labels. Write $\mathbb{E}_{i}$ for expectation with respect to $F_{i}, i=0,1$. The loss function is defined by two increasing functions $U, V$ on $\mathbb{R}$, which yield the objective

$$
C(\alpha, \beta)=-\pi_{1} \mathbb{E}_{1}\left[U\left(\alpha+\beta^{\top} X\right)\right]+\pi_{0} \mathbb{E}_{0}\left[V\left(\alpha+\beta^{\top} X\right)\right]
$$

The first term on the right penalizes small scores in class 1 , and the second term penalizes large scores in class 0 . We can also write this loss function as

$$
C(\alpha, \beta)=\mathbb{E}\left[-Y U\left(\alpha+\beta^{\top} X\right)+(1-Y) V\left(\alpha+\beta^{\top} X\right)\right]
$$

by taking the expectation with respect to the unconditional distribution of $(X, Y)$, under which $\mathbb{P}(Y=i)=\pi_{i}$ and $X$ has distribution $F_{i}$, given $Y=i, i=0,1$.

Let $p(x)=\mathbb{P}(Y=1 \mid X=x)$, and define the log-odds

$$
\eta_{o}(x)=\log \frac{p(x)}{1-p(x)}=\log \frac{\pi_{1}}{\pi_{0}}+\log \frac{d F_{1}}{d F_{0}}(x) \equiv \log \frac{\pi_{1}}{\pi_{0}}+\eta(x) .
$$

The last term $\eta$ is the $\log$ likelihood ratio of $F_{1}$ with respect to $F_{0}$, which is well-defined on the intersection of the support of $F_{0}$ and $F_{1}$. By the Neyman-Pearson lemma, it provides the optimal discriminant function in the sense that it minimizes the error probability $\mathbb{P}_{1}(\eta(X) \leq t)$ 
for any value of the error probability $\mathbb{P}_{0}(\eta(X)>t)$, as $t$ varies. (See, e.g., Theorem 3.2.1 of Lehmann and Romano [13] for a precise statement.) The log-odds $\eta_{o}$ provides an equivalent classifier because it differs from $\eta$ by a constant that can be absorbed in the threshold $t$.

The log-odds need not be linear and thus need not be achievable by minimizing (2). Eguchi and Copas [5] proposed the following restriction on $U$ and $V$ : if the log-odds function is linear, $\eta_{o}(x)=\alpha_{o}+\beta_{o}^{\top} x$, then (2) should be minimized at $\left(\alpha_{o}, \beta_{o}\right)$. In other words, $U$ and $V$ should deliver the optimal classifier if the optimal classifier is linear.

Eguchi and Copas [5] show that this consistency condition holds if the penalty functions $U$ and $V$ satisfy

$$
\frac{\partial V(u)}{\partial u}=e^{u} \frac{\partial U(u)}{\partial u}
$$

equivalently,

$$
U(u)=C_{U}-\int_{u}^{\infty} w(s) d s, \quad V(u)=C_{V}+\int_{-\infty}^{u} e^{s} w(s) d s,
$$

for some positive function $w$ and some constants $C_{U}$ and $C_{V}$. Our analysis applies to linear discriminant functions obtained by minimizing (2) with $U$ and $V$ of this form. The constants $C_{U}$ and $C_{V}$ have no effect in minimizing (2).

The Neyman-Pearson lemma implies that the ROC curve for the log-odds classifier lies above the ROC curve for any other discriminant function. As discussed in Section 2.3 of Eguchi and Copas [5], the loss $C(\alpha, \beta)$ can be interpreted as the weighted area between the log-odds ROC curve and the ROC curve for the linear classifier determined by $(\alpha, \beta)$; the weight assigned to the gap between the curves at a score of $u$ is $w(u)$. By minimizing $C(\alpha, \beta)$, we find the linear score $\alpha+\beta^{\top} x$ that is closest to the true log-odds function, in the sense of this weighted area.

\subsection{Examples of Objective Functions}

With $w(u)=w_{0}(u)=1 /\left(1+e^{u}\right)$, and $C_{U}=C_{V}=0$, we get

$$
U(u)=\log \frac{e^{u}}{1+e^{u}}, \quad V(u)=-\log \frac{1}{1+e^{u}},
$$

and the loss $(2)$ becomes

$$
C(\alpha, \beta)=-\mathbb{E}\left[Y \log \frac{e^{\alpha+\beta^{\top} X}}{1+e^{\alpha+\beta^{\top} X}}+(1-Y) \log \frac{1}{1+e^{\alpha+\beta^{\top} X}}\right] .
$$

Minimizing this expression is equivalent to maximizing the likelihood function in ordinary logistic regression. That is, ordinary logistic regression is a special case of this family of objectives. The discriminant functions $x \mapsto \alpha+\beta^{\top} x$ and $x \mapsto \exp \left(\alpha+\beta^{\top} x\right) /\left(1+\exp \left(\alpha+\beta^{\top} x\right)\right)$ yield equivalent classification rules because each is a monotone transformation of the other. 


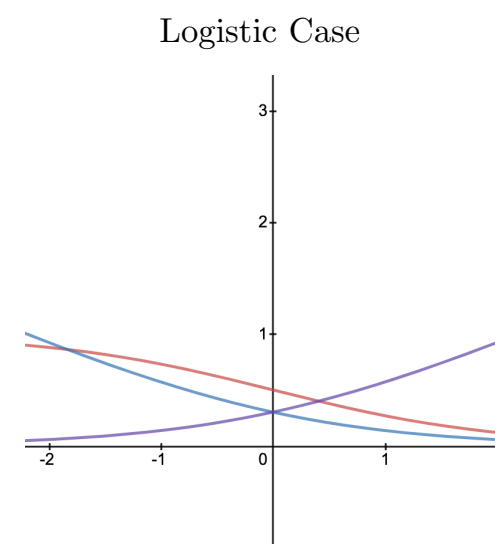

(a) $w(u)=\frac{1}{1+e^{u}}$,
$-U(u)=-\log \frac{e^{u}}{1+e^{u}}, V(u)=-\log \frac{1}{1+e^{u}}$

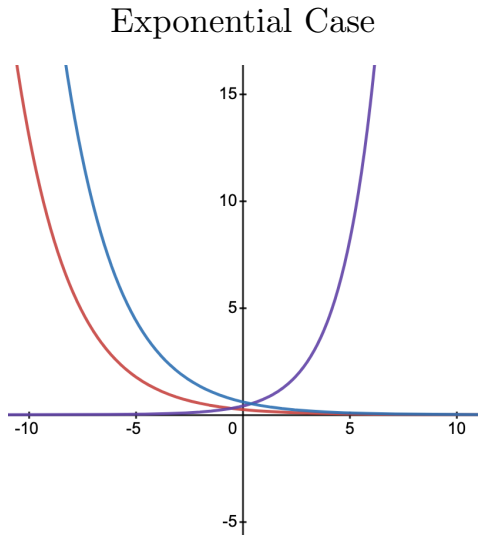

$$
\begin{aligned}
& \text { (b) } w(u)=\lambda(1-\lambda) e^{-\lambda u}, \lambda \in(0,1) \\
& -U(u)=(1-\lambda) e^{-\lambda u}, V(u)=\lambda e^{(1-\lambda) u}
\end{aligned}
$$

Figure 1: Examples of weight functions $w$ and penalty functions $U$ and $V$.

Among other examples, we will also consider exponential weight functions,

$$
w(u)=\lambda(1-\lambda) e^{-\lambda u}, \quad U(u)=-(1-\lambda) e^{-\lambda u}, \quad V(u)=\lambda e^{(1-\lambda) u},
$$

with $\lambda \in(0,1)$, for which the loss function becomes

$$
C(\alpha, \beta)=\mathbb{E}\left[Y(1-\lambda) e^{-\lambda\left(\alpha+\beta^{\top} X\right)}+(1-Y) \lambda e^{(1-\lambda)\left(\alpha+\beta^{\top} X\right)}\right] .
$$

As illustrated in Figure 1, the logistic weight $w_{0}$ is bounded whereas the exponential $w$ is not. A larger $\lambda \in(0,1)$ attaches a greater penalty to large negative values of $\alpha+\beta^{\top} x$ when $y=1$, and a smaller $\lambda \in(0,1)$ attaches a greater penalty to large positive values of $\alpha+\beta^{\top} x$ when $y=0$. Informally, $\lambda$ is balances a trade-off between false negative and false positive probabilities. (We take draws from class 1 to be positive cases.) We will see that a larger $\lambda$ is more sensitive to the distribution of the minority class. The symmetric case $\lambda=1 / 2$ corresponds to the loss function behind the AdaBoost method of Freund and Schapire [9], as discussed in Section 4.1 of Friedman, Hastie, and Tibshirani [7] and Section 2.4 of Eguchi and Copas [5].

\subsection{Empirical Loss}

In estimating $(\alpha, \beta)$, we minimize an empirical version of the loss $(2)$. Let $x_{1}, \ldots, x_{n}$ denote the $n$ observations from class 1 . To capture the idea of extreme imbalance, we adopt the approach in Owen [17]. We assume the number of observations $N$ from class 0 is much larger than $n$, and in fact sufficiently large that we may treat the distribution $F_{0}$ for the majority class as known. We thus arrive at the objective

$$
\bar{C}(\alpha, \beta)=\sum_{i=1}^{n}-U\left(\alpha+\beta^{\top} x_{i}\right)+N \int V\left(\alpha+\beta^{\top} x\right) d F_{0}(x),
$$


which is finite on the set $\Theta$,

$$
\Theta=\{(\alpha, \beta): \bar{V}(\alpha, \beta)<\infty\}, \quad \bar{V}(\alpha, \beta)=\int V\left(\alpha+\beta^{\top} x\right) d F_{0}(x) .
$$

In going from (1) to (7), we replace $\pi_{1}$ with $n /(n+N)$ and $\pi_{0}$ with $N /(n+N)$, and multiply by $n+N$. For fixed $N$, let $\left(\alpha_{N}, \beta_{N}\right)$ minimize (7). We study the behavior of $\left(\alpha_{N}, \beta_{N}\right)$ as $N \rightarrow \infty$.

\section{Existence of a Minimizer}

In this section, we provide conditions ensuring the existence of a unique, finite minimizer of (7). We first state some basic assumptions:

Condition 1 (Basic properties). The weight function $w$ is strictly positive on $\mathbb{R}$. The penalty functions $U$ and $V$ in (4) are well-defined and finite on all of $\mathbb{R}$.

The conditions on $U$ and $V$ imply that $w(s) \rightarrow 0$ and $e^{-s} w(-s) \rightarrow 0$, as $s \rightarrow \infty$. For the loss to be convex, we want $w(u)$ to be decreasing and $e^{u} w(u)$ to be increasing, so we assume

Condition 2 (Convexity). For all $u \in \mathbb{R}, w^{\prime}(u) \leq 0$ and $w(u)+w^{\prime}(u)>0$.

Although weak inequalities would suffice for convexity, we make the second inequality strict to ensure strict convexity, as follows:

Lemma 3.1 (Convex Objective). Under Conditions 1 2, $\bar{C}$ is convex. If $F_{0}$ is not supported on a lower-dimensional subspace of $\mathbb{R}^{d}$, then $\bar{C}$ is strictly convex wherever it is finite.

For ordinary logistic regression, Silvapulle [19] provides necessary and sufficient conditions for the existence of maximum likelihood estimates. These conditions include a requirement of overlap between the two classes. Owen [17] proposes a stronger, but broadly applicable condition to prevent degeneracy. The key idea is that the empirical distribution of the minority class and the true distribution $F_{0}$ for the majority class should overlap at least to the extent that there is probability mass (with respect to $F_{0}$ ) along every possible direction away from the empirical mean of the minority class. We will use this condition as well. It relies on the following definition from Owen [17].

Definition 1 (Surrounding property). The distribution $F$ on $\mathbb{R}^{d}$ surrounds the point $x^{*}$ if for some $\epsilon>0$, for some $\delta>0$ and for all $\omega \in \Omega=\left\{\omega \in \mathbb{R}^{d} \mid \omega^{\top} \omega=1\right\}$

$$
\int_{\left(x-x^{*}\right)^{\top} \omega>\epsilon} d F(x)>\delta .
$$

Condition 3 (Surrounding minority mean). $F_{0}$ surrounds $\bar{x}$, where $\bar{x}=\left(x_{1}+\cdots+x_{n}\right) / n$ is the mean of the minority class. 
If $F_{0}$ surrounds a point in $\mathbb{R}^{d}$, it satisfies the condition in Lemma 3.1. We impose the following regularity condition to ensure $\bar{V}$ in $(8)$ grows smoothly near the boundary of $\Theta$ :

Condition 4 (Semincontinuity). $\bar{V}$ is lower semicontinuous on $\mathbb{R}^{d+1}$, and $\Theta$ is open.

Lemma 3.2 (Existence). Let $N, n \geq 1$ and $x_{1}, \ldots, x_{n} \in \mathbb{R}^{d}$ be given. If Conditions 14 hold and $F_{0}$ has finite mean, then the loss function $\bar{C}(\alpha, \beta)$ in $(7)$ has a unique finite minimizer $\left(\alpha_{N}, \beta_{N}\right)$.

\section{Convergence Under Infinite Imbalance}

We now come to our main results, identifying the behavior of the optimal $\left(\alpha_{N}, \beta_{N}\right)$ in the limit of infinite imbalance. Our analysis considers three classes of weight functions $w(u)-$ bounded, asymptotically exponential, and asymptotically polynomial — based primarily on their properties for large negative values of $u$. We use the relation $\sim$ to indicate that the ratio of two functions converges to 1 .

Definition 2. We consider the following types of weight functions w:

- $w(u)$ is bounded if $w(u) \leq C$ for all $u$, for some $C>0$;

- $w(u)$ is asymptotically polynomial if

- the left tail of $w$ is polynomial, in the sense that $w(u) \sim C|u|^{k}$, as $u \rightarrow-\infty$, for some $C>0$, and $k>0$, and

- the right tail of $w(u)$ satisfies $\lim \sup _{u \rightarrow \infty} V(u) / e^{u} w(u)<\infty$.

- $w(u)$ is asymptotically exponential if

- the left tail of $w$ is exponential, in the sense that $w(u) \sim C e^{-\lambda u}$, as $u \rightarrow-\infty$, for some $C>0$, and $\lambda \in(0,1)$, and

- $w$ is everywhere exponentially lower-bounded, in the sense that $w(u) \geq C_{L} e^{-\lambda_{1} u}$, for all $u \in \mathbb{R}$, for some $\lambda_{1} \in(0,1)$ and some $C_{L}>0$;

Our focus in this categorization is on the left-tail behavior of the weight function $w$ because under infinite imbalance we expect $\alpha_{N} \rightarrow-\infty$; this limit is suggested by letting $\pi_{1} \rightarrow 0$ in (3). The additional conditions help ensure that $\alpha_{N}$ indeed diverges and $\beta_{N}$ remains bounded. The example of logistic regression (5) considered by Owen [17] is covered by the bounded case. The right tail condition in the asymptotically polynomial case is satisfied if $e^{u} w(u)$ is log-convex on some interval $\left[u_{0}, \infty\right)$, and this condition is satisfied if $w(u)=C e^{-\lambda_{1} u}, \lambda_{1} \in(0,1)$, or 
$w(u)=C u^{-k}, k>0$, for large $u$; see Section B.3 of the appendix for a more general result. Although we use the term "polynomial" in the last category of weight functions, we do not require $k$ to be an integer. The following lemma is the key step in our analysis.

Lemma 4.1. Suppose Conditions 14 hold and $F_{0}$ has finite mean.

(i) If $w$ is bounded or asymptotically exponential, then

$$
\alpha_{N} \rightarrow-\infty, \quad \limsup _{N \rightarrow \infty}\left\|\beta_{N}\right\|<\infty
$$

(ii) If $w$ is asymptotically polynomial and $F_{0}$ surrounds $x_{1}, \ldots, x_{n}$, then (10) holds.

Under the conditions of Lemma 4.1, $\beta_{N}$ is contained in a closed ball of radius $r$, for all sufficiently large $N$. A valid radius $r$ is given explicitly in the proof of Lemma B.2 in the appendix. For our main results, we require the following tail condition on the majority class distribution $F_{0}$ :

Condition 5 (Tail condition).

$$
\int e^{r\|x\|}\left(1+\|x\|^{k+1}\right) d F_{0}(x)<\infty,
$$

where $k=0$ in the case of bounded or asymptotically exponential $w$.

This condition is satisfied by distributions $F_{0}$ with bounded support, with Gaussian tails, or with tails that decay exponentially at a rate faster than $r$.

Armed with 10 and (11), we can now show that $\beta_{N}$ converges under infinite imbalance. Bounded and asymptotically polynomial weight functions lead to the same limiting coefficient vector; in the asymptotically exponential case, the limit depends on the exponent $\lambda$.

Theorem 4.2 (Bounded or Asymptotically Polynomial w). Suppose Conditions 15 hold. Suppose that (i) $w$ is bounded or (ii) $w$ is asymptotically polynomial and $F_{0}$ surrounds $x_{1}, \ldots, x_{n}$. Then the minimizer $\left(\alpha_{N}, \beta_{N}\right)$ of $\bar{C}$ in (भ) satisfies $\alpha_{N} \rightarrow-\infty$ and $\beta_{N} \rightarrow \beta_{*}$, where $\beta_{*}$ is the unique solution to

$$
\frac{\int e^{\beta_{*}^{\top} x} x d F_{0}(x)}{\int e^{\beta_{*}^{\top} x} d F_{0}(x)}=\bar{x} .
$$

Theorem 4.3 (Asymptotically Exponential $w$ ). Suppose that $w$ is asymptotically exponential and that Conditions 15 hold, with $k=0$ in (11). Then the minimizer $\left(\alpha_{N}, \beta_{N}\right)$ of $\bar{C}$ in (7) satisfies $\alpha_{N} \rightarrow-\infty$ and $\beta_{N} \rightarrow \beta_{*}$, where $\beta_{*}$ is the unique solution to

$$
\frac{\int x e^{(1-\lambda) \beta_{*}^{\top} x} d F_{0}(x)}{\int e^{(1-\lambda) \beta_{*}^{\top} x} d F_{0}(x)}=\frac{\sum_{i=1}^{n} x_{i} e^{-\lambda \beta_{*}^{\top} x_{i}}}{\sum_{i=1}^{n} e^{-\lambda \beta_{*}^{\top} x_{i}}} .
$$


Theorem 4.2 shows, in particular, that a broad class of linear discriminant functions are asymptotically equivalent to logistic regression under infinite imbalance in that they have a common limiting coefficient vector. Theorem 4.3 allows a wider range of limits. The conclusion of Theorem 4.3 can be strengthened if the weight function $w$ is not just asymptotically exponential but in fact exactly exponential, in the sense that

$$
w(u)=C e^{-\lambda u}, \text { for all } u \in \mathbb{R} \text {, for some } C>0 \text { and } 0<\lambda<1 \text {. }
$$

With this choice of $w$, Conditions 1 and 2 are satisfied. The optimal $\beta_{N}$ satisfies $(13)$ for all $N$, not just in the limit:

Theorem 4.4 (Exactly Exponential $w$ ). Suppose $w$ has the form in (14). Suppose Conditions $3 \sqrt{5}$ hold, with $k=0$ in (11). Then the minimizer $\left(\alpha_{N}, \beta_{N}\right)$ of $\bar{C}$ in (7) satisfies $\alpha_{N} \rightarrow-\infty$ and $\beta_{N} \equiv \beta_{*}$, where $\beta_{*}$ is the unique solution to 13 .

To show that not all choices of $w$ lead to similar limits, we briefly consider a case suggested in Section 2.4 of Eguchi and Copas [5]. Taking $w$ to be a delta function with unit mass at a point $u_{0}$, they arrive at the objective

$$
C(\alpha, \beta)=\pi_{1} \mathbb{P}_{1}\left(\alpha+\beta^{\top} X \leq u_{0}\right)+\pi_{0} e^{u_{0}} \mathbb{P}_{0}\left(\alpha+\beta^{\top} X>u_{0}\right) .
$$

Through appropriate choice of $u_{0}, C(\alpha, \beta)$ can be interpreted as balancing misclassification costs. However, we show in Appendix $\mathrm{D}$ that the resulting discriminant function degenerates under imbalance, in the sense that for all sufficiently large $N, \beta_{N}=0$ and $\alpha_{N}$ can be any value less than or equal to $u_{0}$.

Li, Belloti, and Adams [14] consider regularized logistic regression with an $L_{1}$ or $L_{2}$ penalty on $\beta$. They show that the optimal $\beta_{N}$ converges to zero under infinite imbalance. In this sense, the regularized discriminant function degenerates under imbalance.

\section{Robustness Interpretation of $\beta_{*}$}

We now turn to the interpretation of the limits $\beta_{*}$ defined by 12 and 13 . We will show that these limits reflect robustness properties, in the sense that the coefficients are optimized against certain worst-case errors or combinations of errors. These robustness properties reflect implicit choices of conservatism towards different types of errors.

\subsection{Bounded and Asymptotically Polynomial Case}

The robustness interpretation is easiest to formulate in the setting of Theorem 4.2 , for bounded and asymptotically polynomial $w$. For $\beta \in \mathbb{R}^{d}$, define the cumulant generating function of $F_{0}$ 
by setting

$$
\psi(\beta)=\log \int e^{\beta^{\top} x} d F_{0}(x),
$$

and let $B_{\psi}=\{\beta: \psi(\beta)<\infty\}$. Define the exponential family of distributions $F_{\beta}, \beta \in B_{\psi}$, on $\mathbb{R}^{d}$ by setting

$$
d F_{\beta}(x)=e^{\beta^{\top} x-\psi(\beta)} d F_{0}(x) .
$$

Part of the content of Theorem 4.2 is that $\beta_{*} \in B_{\psi}$; write $F_{*}$ for the special case $F_{\beta_{*}}$. Equation (12) tells us that

$$
\int x d F_{*}(x)=\int e^{\beta_{*}^{\top} x-\psi(\beta)} d F_{0}(x)=\bar{x}
$$

the mean of $F_{*}$ is $\bar{x}$.

Let $G$ be any distribution on $\mathbb{R}^{d}$ with same support as $F_{0}$. As discussed in Section 2 , the Neyman-Pearson lemma shows that optimal rules for discriminating between $G$ and $F_{0}$ use the $\log$ likelihood ratio, $\eta(x)=\log d G(x) / d F_{0}(x)$. One way to describe the optimality of $\eta$ is through the ROC curve, which is the set of points given by coordinates $\left(\mathbb{P}_{F_{0}}(\eta(X)>t), \mathbb{P}_{G}(\eta(X)>t)\right)$, $-\infty<t<\infty$. (The subscript on $\mathbb{P}$ indicates the distribution of $X$.) The second coordinate is the sensitivity of the classifier, and the first is 1 minus the specificity. The ROC curve defined by the log likelihood ratio $\eta$ lies above the ROC curve for any other discriminant function.

In the case of the distribution $F_{*}$ defined by $\beta_{*}$, the log likehoood ratio is given by

$$
\eta_{*}(x)=\log \frac{d F_{*}}{d F_{0}}(x)=\beta_{*}^{\top} x-\psi\left(\beta_{*}\right) .
$$

The linear discriminant function $\beta_{*}^{\top} x$ coincides with the optimal classifier $\eta_{*}$. The constant $\psi\left(\beta_{*}\right)$ shifts the threshold $t$, but the two functions trace the same ROC curve. In other words, the limit in 12 picks the optimal classifier for discriminating between $F_{0}$ and $F_{*}$.

The robustness interpretation comes from identifying $F_{*}$ as a worst-case alternative. For distributions $G$ and $F$ on $\mathbb{R}^{d}$, define the relative entropy (or Kullback-Leibler divergence),

$$
D(G \| F)=\int \log \frac{d G}{d F} d G
$$

with $D(G \| F)=\infty$ if the support of $G$ is not contained within the support of $F$. Relative entropy is always non-negative, and it is zero if and only if $G$ and $F$ coincide. It is not symmetric, but $D(G \| F)$ can be interpreted as a measure of the "distance" of $G$ from $F$. If $D(G \| F)$ is small then $G$ is close to $F$, making the problem of discriminating between the two distributions difficult. Kullback and Leibler [12] interpret $D(G \| F)$ as the mean information for discriminating between $G$ and $F$ per observation from $G$.

Let $\mathcal{M}_{\bar{x}}$ be the set of probability distributions on $\mathbb{R}^{d}$ with mean $\bar{x}$. We know from 17 that $F_{*} \in \mathcal{M}_{\bar{x}}$. In fact, of all elements of $\mathcal{M}_{\bar{x}}$, the one "closest" to $F_{0}$ in the sense of relative entropy is $F_{*}$, as the following result shows. This result is a special case of Corollary 3.1 of Csiszar [3]. 
Lemma 5.1. For all $G \in \mathcal{M}_{\bar{x}}$,

$$
D\left(G \| F_{0}\right)=D\left(G \| F_{*}\right)+D\left(F_{*} \| F_{0}\right) .
$$

Thus, $D\left(F_{*} \| F_{0}\right)=\min _{G \in \mathcal{M}_{\bar{x}}} D\left(G \| F_{0}\right)$.

Combining this lemma with (18), we arrive at the following conclusion: The limiting coefficient $\beta_{*}$ in 12 provides the optimal classification rule for the worst-case alternative to the majority class distribution $F_{0}$, among all distributions with same mean $\bar{x}$ as the observations from the minority class. The distribution $F_{*}$ presents the worst case because it is hardest to distinguish from $F_{0}$.

The definition in (19) suggests an interpretation of the conservatism implicit in the choice of $F_{*}$. In making $D\left(G \| F_{0}\right)$ smaller, we are, roughly speaking, making the optimal discriminant function (the $\log$ likelihood ratio $\log \left(d G / d F_{0}\right)$ ) smaller at observations that have higher probability under $G$. In other words, we are focusing on a case in which the optimal classifier will have low sensitivity. This perspective suggests that the limiting coefficient $\beta_{*}$ in 12 is implicitly conservative in classifying to the minority class and may perform better at highspecificity thresholds than at high-sensitivity thresholds. This interpretation will be supported by our discussion of exponential objectives in Section 5.4 and the numerical results of Sections 6 and 7 .

\subsection{Infinite Upsampling}

The problem of imbalance is sometimes dealt with in practice through upsampling — creating artificial data from the minority class. We now show that for the class of linear discriminant functions defined by minimizing (1) with bounded or asymptotically polynomial $w$ (including logistic regression), the estimate of $\beta$ obtained in the infinite imbalance limit coincides with the estimate obtained from a specific choice of upsampling distribution, namely $F_{*}$.

Suppose, then, that we have infinitely upsampled the minority class so that a fraction $\pi_{1}$ of our data is drawn from $F_{*}$, leading to a loss function of the form (1). Differentiating (1) with respect to $\beta$ and recalling that $U^{\prime}(s)=w(s), V^{\prime}(s)=e^{s} w(s)$, we get the first-order condition

$$
-\pi_{1} \mathbb{E}_{1}\left[w\left(\alpha+\beta^{\top} x\right) x\right]+\pi_{0} \mathbb{E}_{0}\left[e^{\alpha+\beta^{\top} x} w\left(\alpha+\beta^{\top} x\right)\right]=0,
$$

where $\mathbb{E}_{1}$ is now expectation with respect to $F_{*}$. Using the likelihood ratio $d F_{*} / d F_{0}$, as in (18), we can write the first term as an expectation with respect to $F_{0}$ to get

$$
-\pi_{1} \mathbb{E}_{0}\left[e^{\beta_{*}^{\top} x-\psi\left(\beta_{*}\right)} w\left(\alpha+\beta^{\top} x\right) x\right]+\pi_{0} \mathbb{E}_{0}\left[e^{\alpha+\beta^{\top} x} w\left(\alpha+\beta^{\top} x\right) x\right]=0 .
$$


This equation is solved by taking

$$
\beta=\beta_{*}, \quad e^{\alpha}=\left(\pi_{1} / \pi_{0}\right) e^{-\psi\left(\beta_{*}\right)} .
$$

The limiting coefficient vector $\beta_{*}$ identified in Theorem 4.2 is thus precisely what one would obtain through infinite upsampling of the minority class using $F_{*}$. The degree of upsampling, as reflected in $\pi_{1} / \pi_{0}$, affects $\alpha$ but not $\beta$.

The choice of $F_{*}$ as an upsampling distribution is not arbitrary. We know from Section 5.1 that $F_{*}$ is the distribution "closest" to $F_{0}$ among all distributions with the mean $\bar{x}$ in the data from the minority class. In this sense, upsampling according to $F_{*}$ is conservative if one is concerned about overstating the sensitivity of a classification rule in identifying draws from the minority class.

\subsection{The Gaussian Case}

The Gaussian case provides some convenient simplifications and helps illustrate the transition from the limit in $(12)$ to the limit in $(13)$. For this discussion, we briefly leave aside the question of imbalance and let $F_{i}$, be multivariate normal distributions $N\left(\mu_{i}, \Sigma\right)$ with means $\mu_{i}, i=0,1$, and a common full-rank covariance matrix $\Sigma$. Classical linear discriminant analysis provides the coefficient vector

$$
\beta_{*}=\Sigma^{-1}\left(\mu_{1}-\mu_{0}\right)
$$

observations $x$ are then classified to class 1 or 0 depending on whether $\beta_{*}^{\top} x$ is larger or smaller than some threshold. We ignore the intercept, which can be absorbed into the choice of threshold.

This choice of coefficient vector coincides with 12 in the sense that if we replace $\beta_{*}$ in $(12)$ with $\Sigma^{-1}\left(\mu_{1}-\mu_{0}\right)$ when $F_{0}$ is $N\left(\mu_{0}, \Sigma\right)$, we get $\mu_{1}$. In other words, when $F_{0}$ is multivariate normal, the infinite imbalance limit in Theorem 4.2 is what one would get by assuming $F_{1}$ is multivariate normal with the same covariance matrix. This is a special case of the upsampling interpretation in Section 5.2 , because Gaussian distributions with a common covariance matrix form an exponential family.

In the left panel of Figure 2, the ellipses show probability contours for two bivariate normal distributions $N\left(\mu_{i}, \Sigma_{i}\right), i=0,1$, with different covariance matrices and different means. Class 0 is indicated in blue, and class 1 in red. The blue line illustrates the vector $\beta_{*}=\Sigma_{0}^{-1}\left(\mu_{1}-\mu_{0}\right)$, the discriminant computed using the covariance matrix from the blue distribution; the red line illustrates the corresponding line $\Sigma_{1}^{-1}\left(\mu_{1}-\mu_{0}\right)$ calculated from the perspective of the red distribution. (We have flipped the sign so that in both cases larger values of $\beta_{*}^{\top}$ are more indicative of values from the red distribution.) The intercepts of the lines are arbitrary; the 

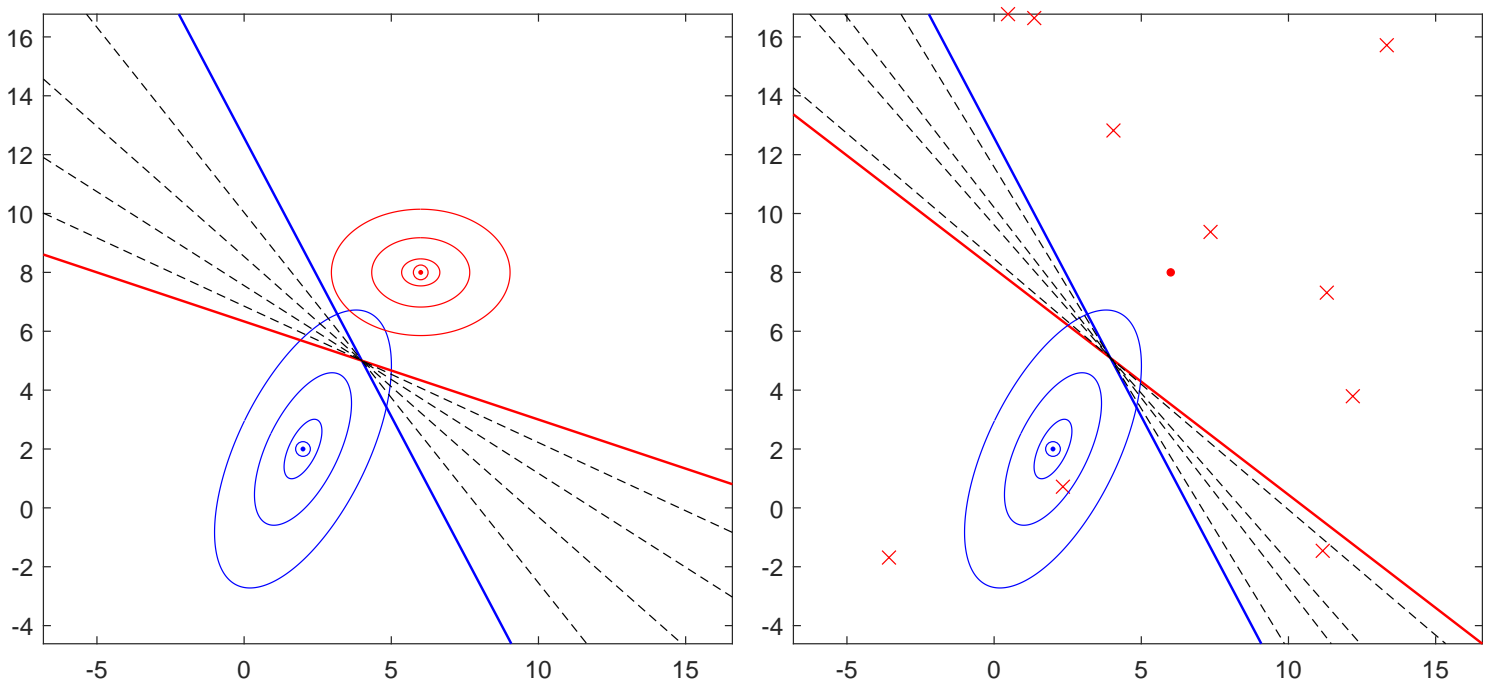

Figure 2: Classification boundaries with unequal covariance matrices. Dashed lines show the effect of varying $\lambda$.

classification rules should be thought of as sets of lines parallel to the blue or red line, with different intercepts corresponding to different classification thresholds. The slopes of the lines differ because (building on the discussion on Section 5.1), the blue line is optimized against $N\left(\mu_{1}, \Sigma_{0}\right)$, and the red line is optimized against $N\left(\mu_{0}, \Sigma_{1}\right)$.

Now consider the analog of $(13)$ in which the distributions on the two sides $\left(F_{0}\right.$ on the left, the empirical distribution on $x_{1}, \ldots, x_{n}$ on the right) are replaced with normal distributions. In this case 13 reduces to

$$
\mu_{0}+(1-\lambda) \Sigma_{0} \beta_{*}=\mu_{1}-\lambda \Sigma_{1} \beta_{*}
$$

i.e.,

$$
\beta_{*}=\left(\lambda \Sigma_{1}+(1-\lambda) \Sigma_{0}\right)^{-1}\left(\mu_{1}-\mu_{0}\right) .
$$

Thus, the exponent $\lambda$ in the asymptotically exponential weight function $w$ balances the relative influence of the two distributions in setting the slope of the classifier. The dashed lines in the left panel of Figure 2 show the effect of varying $\lambda$ between 0 and 1 .

The extremes $\lambda=0$ and $\lambda=1$ correspond to two perspectives on conservatism, through the interpretation in Section 5.1. We noted there that $\lambda=0$ focuses on worst-case sensitivity in detecting samples from $F_{1}$; similarly, $\lambda=1$ focuses on worst-case specificity. We return to these points in the examples of Sections 6 and 7 .

Now replace the normal distribution for $F_{1}$ with the empirical distribution on $x_{1}, \ldots, x_{n}$, 
while keeping $F_{0}$ normal. In this case 13 becomes

$$
\mu_{0}+(1-\lambda) \Sigma_{0} \beta_{*}=\frac{\sum_{i=1}^{n} x_{i} e^{-\lambda \beta_{*}^{\top} x_{i}}}{\sum_{i=1}^{n} e^{-\lambda \beta_{*}^{\top} x_{i}}},
$$

which can be solved numerically. The crosses in the right panel of Figure 2 represent $x_{1}, \ldots, x_{n}$. Their mean is at $(6,8)$, just as it is for the red normal distribution in the left panel. The slopes of the solid blue lines are therefore the same in the two panels: the discriminant computed from the blue distribution $(\lambda=0)$ depends on the red distribution only through its mean. The solid red line $(\lambda=1)$ similarly depends on $F_{0}$ only through $\mu_{0}$.

The dashed black lines correspond to intermediate values of $\lambda$. These make use of the distributions of both classes, not just their means, indicating a potential advantage of the exponential objective over logistic regression and other bounded or asymptotically polynomial cases. Although it is not evident from the figure, the slopes of the lines need not change monotonically with $\lambda$, nor is the slope at an intermediate $\lambda$ necessarily between the slopes of the blue and red lines.

\subsection{Asymptotically Exponential Case}

We now extend the robustness property of Lemma 5.1 to the setting of (13). For distributions $F_{0}, F_{1}$ on $\mathbb{R}^{d}$, define the cumulant generating functions $\psi_{i}, i=0,1$, as in 16 , with domains $B_{i}, i=0,1$, and define the exponential families of distributions

$$
d F_{i, \beta}(x)=e^{\beta^{\top} x-\psi_{i}(\beta)} d F_{i}(x), \quad \beta \in B_{i} .
$$

Proposition 5.1. For $\lambda \in(0,1)$, suppose there is a $\beta_{*} \in B_{0} \cap B_{1}$ for which

$$
\nabla \psi_{0}\left((1-\lambda) \beta_{*}\right)=\nabla \psi_{1}\left(-\lambda \beta_{*}\right)
$$

Then the problem

$$
\min _{G_{0}, G_{1}} \lambda D\left(G_{0} \| F_{0}\right)+(1-\lambda) D\left(G_{1} \| F_{1}\right) \text { subject to } \int x d G_{0}(x)=\int x d G_{1}(x),
$$

where $G_{0}$ and $G_{1}$ are distributions on $\mathbb{R}^{d}$, is solved by $G_{0}=F_{0,(1-\lambda) \beta_{*}}$ and $G_{1}=F_{1,-\lambda \beta_{*}}$.

Proof. For any value $\mu$ of the common mean required by the constraint in 22 , we know from Lemma 5.1 that $D\left(G_{i} \| F_{i}\right), i=0,1$, is minimized by taking $G_{i}$ to be an exponentially tilted distribution $F_{i, \beta_{i}}$, with $\nabla \psi_{i}\left(\beta_{i}\right)=\mu, i=0,1$. We then get

$$
D\left(G_{i} \| F_{i}\right)=\int \beta_{i}^{\top} x-\psi_{i}\left(\beta_{i}\right) d G_{i}=\beta_{i}^{\top} \mu-\psi_{i}\left(\beta_{i}\right), \quad i=0,1,
$$


and an objective function value of

$$
\lambda\left[\beta_{0} \mu-\psi_{0}\left(\beta_{0}\right)\right]+(1-\lambda)\left[\beta_{1} \mu-\psi_{1}\left(\beta_{1}\right)\right]
$$

We can solve (22) by minimizing (23) over $\mu$, keeping in mind that $\beta_{0}$ and $\beta_{1}$ depend on $\mu$.

Each function $\mu \mapsto \beta_{i} \mu-\psi_{i}\left(\beta_{i}\right)$ in $(23)$ is the convex conjugate of $\psi_{i}, i=0,1$, at $\mu$, defined by

$$
\sup _{b}\left\{b \mu-\psi_{i}(b)\right\}
$$

and is therefore convex in $\mu$. It follows that any point at which the derivative of (23) with respect to $\mu$ is zero minimizes 23 .

Differentiating 23 with respect to $\mu$, writing $\dot{\beta}_{i}$ for the derivative matrix of $\beta_{i}$ with respect to $\mu$, and setting the derivative equal to zero to get

$$
\lambda\left[\dot{\beta}_{0} \cdot \mu+\beta_{0}-\dot{\beta}_{0} \cdot \nabla \psi_{0}\left(\beta_{0}\right)\right]+(1-\lambda)\left[\dot{\beta}_{1} \cdot \mu+\beta_{1}-\dot{\beta}_{1} \cdot \nabla \psi_{1}\left(\beta_{1}\right)\right]=0 .
$$

But $\nabla \psi_{i}\left(\beta_{i}\right)=\mu, i=0,1$, so this equation simplifies to

$$
\lambda \beta_{0}+(1-\lambda) \beta_{1}=0 .
$$

The solution is then of the form $\beta_{0}=(1-\lambda) \beta$ and $\beta_{1}=-\lambda \beta$, where $\beta$ solves

$$
\nabla \psi_{0}((1-\lambda) \beta)=\nabla \psi_{1}(-\lambda \beta)
$$

which is 21.

Equation (21) generalizes (13); it reduces to 13 when $F_{1}$ is the empirical distribution on $x_{1}, \ldots, x_{n}$. The limiting coefficient vector in (13) can therefore be interpreted as the result of minimizing the objective in $(22)$, with this substitution for $F_{1}$. Whereas $(12)$, or Lemma 5.1 , implicitly focuses on the worst-case distribution from the perspective of $F_{0}$, the objective in 22 balances the worst case as seen from both $F_{0}$ and $F_{1}$. It thus balances conservatism toward sensitivity and specificity.

To understand this balance, recall from the discussion at the end of Section 2.1 that the weight function $w(u)$ can be interpreted as a penalty on the difference between the ROC curves for the optimal discriminant function and an approximating linear discriminant. With $w(u)$ proportional to $e^{-\lambda u}$, we thus expect a better approximation at large negative $u$ (high sensitivity) when $\lambda$ is close to 1 , and a better approximation at large positive $u$ (high specificity) when $\lambda$ is close to 0 . This pattern is what we find in the experiments of Sections 6 and 7.

We can gain further insight into the connection between $(22)$ and the original classification problem by generalizing (6) to the problem of minimizing

$$
\bar{C}_{\lambda}(R)=\mathbb{E}\left[Y(1-\lambda) e^{-\lambda R(X)}+(1-Y) \lambda e^{(1-\lambda) R(X)}\right]
$$


over possibly nonlinear discriminant functions $R: \mathbb{R}^{d} \rightarrow \mathbb{R}$. Suppose $F_{0}$ and $F_{1}$ have densities $f_{0}$ and $f_{1}$. Then arguing as in Lemma 1 of Friedman, Hastie, and Tibshirani [7, 24] is minimized at

$$
R(x)=\log \frac{\pi_{1}}{\pi_{0}}+\log \frac{f_{1}(x)}{f_{2}(x)} .
$$

Making this substitution in 24) and simplifying yields

$$
\bar{C}_{\lambda}(R)=\int\left(\pi_{1} f_{1}(x)\right)^{1-\lambda}\left(\pi_{0} f_{0}(x)\right)^{\lambda} d x .
$$

The case $\lambda=1 / 2$ appears in equation (28) of Eguchi and Copas [5]. We can write 25] as

$$
\bar{C}_{\lambda}(R)=\pi_{1}^{1-\lambda} \pi_{0}^{\lambda} e^{(\lambda-1) D_{\lambda}\left(F_{0} \| F_{1}\right)},
$$

using the Rényi divergence,

$$
D_{\lambda}(H \| F)=\frac{1}{\lambda-1} \log \int d H^{\lambda} d F^{(1-\lambda)} .
$$

The Rényi divergence has a representation in terms of the Kullback-Leibler divergence as

$$
(1-\lambda) D_{\lambda}(H \| F)=\inf _{G}\{\lambda D(G \| H)+(1-\lambda) D(G \| F)\}
$$

(Erven and Harremoës [6], Theorem 30) which allows us to write 26) as

$$
\bar{C}_{\lambda}(R)=\pi_{1}^{1-\lambda} \pi_{0}^{\lambda} e^{-\inf _{G}\left\{\lambda D\left(G \| F_{0}\right)+(1-\lambda) D\left(G \| F_{1}\right)\right\}} .
$$

In other words, when we drop the requirement that the discriminant function be linear, the minimal loss (24) is determined by

$$
\inf _{G}\left\{\lambda D\left(G \| F_{0}\right)+(1-\lambda) D\left(G \| F_{1}\right) .\right.
$$

The loss in (27) is small when there is no $G$ that is "close" to both $F_{0}$ and $F_{1}$ in the sense of this weighted divergence. When we impose the linearity constraint, the optimal $\beta$ is determind by (22), which relaxes the constraint that $G_{0}=G_{1}$ to the requirement that these two distributions have the same mean.

The symmetric case $\lambda=1 / 2$ admits a further interpretation. For any discriminant function, the area under the curve measure AUC, discussed further in the next section, equals the probability that a draw $X_{1}$ from $F_{1}$ scores higher than an independent draw $X_{0}$ from $F_{0}$. Thus, for a linear discriminant function $x \mapsto \beta^{\top} x$, Markov's inequality yields

$$
\mathrm{AUC}=1-\mathbb{P}\left(\beta^{\top} X_{0} \geq \beta^{\top} X_{1}\right) \geq 1-e^{\psi_{0}\left(\frac{1}{2} \beta\right)+\psi_{1}\left(-\frac{1}{2} \beta\right)} .
$$

Maximizing the lower bound over $\beta$ leads to the first-order condition $\nabla \psi_{0}(\beta / 2)=\nabla \psi_{1}(-\beta / 2)$, which is the condition in 21) with $\lambda=1 / 2$. This observation is consistent with the idea that taking $\lambda=1 / 2$ balances overall performance without emphasizing either specificity or sensitivity over the other. 


\section{$6 \quad$ Numerical Examples}

We use simulations to examine the convergence of $\beta_{N}$ and to illustrate properties of the classifiers derived using various choices of the penalty function $w$.

\subsection{Convergence Simulations}

For simplicity, we examine convergence in a one-dimensional example. We have just two observations from the minority class, $x_{1}=0$ and $x_{2}=1$. For the majority class, we take $F_{0}$ to be the standard normal distribution, $N(0,1)$. To get an accurate approximation to $F_{0}$, we use a stratified sample in which $x_{i}$ is the $\frac{i-0.5}{N}$-quantile of $N(0,1)$, for $i=1, \ldots, N$. We compare results at several values of $N$.

Table 1 reports the regression coefficients $\left(\alpha_{N}, \beta_{N}\right)$ for ordinary logistic regression, two exponential objectives, and an asymptotically linear objective. For the exponential objectives we consider $\lambda=0.1$ and $\lambda=0.9$, and for the asymptotically linear objective we use $w(u)=$ $-2 u+1$, for $u \leq 0$, and $w(u)=(u+1)^{-2}$ for $u>0$.

We solve for the optimal coefficients using the minimize function in the scipy.optimizer package with the BFGS optimization method. The last row of Table 1 reports the limiting value $\beta_{*}$ determined by Theorems 4.2 and 4.4 , calculated by solving 12 and 13 .

\begin{tabular}{c|rc|rc|rr|rr}
\hline & \multicolumn{2}{|c|}{ Logistic $w$} & \multicolumn{2}{|c|}{$\lambda=0.1$} & \multicolumn{2}{|c|}{$\lambda=0.9$} & \multicolumn{2}{|c}{ Linear $w$} \\
$N$ & $\alpha_{N}$ & $\beta_{N}$ & $\alpha_{N}$ & $\beta_{N}$ & $\alpha_{N}$ & $\beta_{N}$ & $\alpha_{N}$ & $\beta_{N}$ \\
\hline 10 & -1.78 & 0.67 & -1.78 & 0.63 & -2.14 & 1.84 & -1.85 & 0.95 \\
100 & -4.04 & 0.52 & -4.06 & 0.55 & -4.43 & 1.75 & -4.08 & 0.62 \\
1,000 & -6.34 & 0.50 & -6.36 & 0.54 & -6.73 & 1.74 & -6.37 & 0.57 \\
10,000 & -8.64 & 0.50 & -8.66 & 0.54 & -9.03 & 1.73 & -8.66 & 0.55 \\
100,000 & -10.94 & 0.50 & -10.96 & 0.54 & -11.34 & 1.73 & -10.96 & 0.54 \\
\hline True $\beta_{*}$ & & 0.50 & & 0.54 & & 1.73 & & 0.50 \\
\hline
\end{tabular}

Table 1: Convergence of coefficients as the sample size $N$ of the majority class grows.

In all cases, we see that $\alpha_{N} \rightarrow-\infty$ at rate $\log N$. For logistic regression and the exponential objectives, $\beta_{N}$ is close to $\beta_{*}$ at $N=100$, when the minority class constitutes $2 \%$ of the data. For these cases, the infinite-imbalance limit provides a very good approximation even without extreme imbalance. In the asymptotically linear case, we know from Theorem 4.2 that $\beta_{N}$ approaches the same limit $\beta_{*}$ as in the case of logistic regression, but the results in the table indicate that the convergence is much slower. We have observed the same behavior in other examples. This pattern suggests that the case of polynomial $w$ is qualitatively different from the bounded and exponential cases, even though it has the same limit as the bounded case. 


\subsection{High-Sensitivity and High-Specificity Regions}

We turn next to a comparison of logistic regression with exponential objectives at various values of the exponent $\lambda$. As we discussed in Sections 2.2 and 5.4 , we expect the value of $\lambda$ to control the relative performance of a classifier as measured by sensitivity or specificity.

We consider a two-dimensional example in which $F_{0}$ is the bivariate standard normal distribution. Samples from the minority class are drawn from a mixture of two normals: we have a sample of $n=500$, of which $10 \%$ are drawn from $N\left(\mu_{1,1}, \Sigma_{1,1}\right)$ and $90 \%$ from $N\left(\mu_{1,2}, \Sigma_{1,2}\right)$, with

$$
\mu_{1,1}=\left(\begin{array}{l}
0 \\
2
\end{array}\right), \Sigma_{1,1}=\left(\begin{array}{cc}
0.3 & 0 \\
0 & 0.3
\end{array}\right), \quad \mu_{1,2}=\left(\begin{array}{l}
2.3 \\
2.3
\end{array}\right), \Sigma_{1,2}=\left(\begin{array}{cc}
0.2 & 0 \\
0 & 0.2
\end{array}\right) .
$$

We will compare results at various values of the sample size $N$ for the majority class.

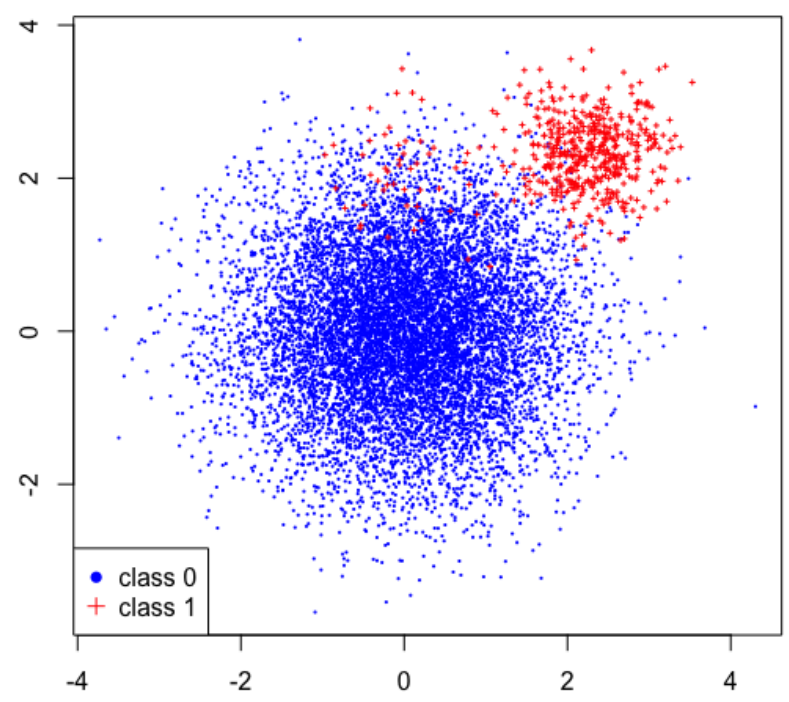

Figure 3: Two-Dimensional Example

Figure 3 shows points drawn from the two classes, with $N=10,000$ and $n=500$. The example is designed so that $90 \%$ of the minority class comes from a distribution that is easily distinguishable from the majority class, but the remaining $10 \%$ make the classification task challenging.

Alternative classifiers are often compared through their ROC curves. Recall that the ROC curve for a linear discriminant function $\beta^{\top} x$ is a plot of the true positive rate $\mathbb{P}_{1}\left(\beta^{\top} X>\right.$ t) against the false positive rate $\mathbb{P}_{0}\left(\beta^{\top} X>t\right)$, for all $t \in \mathbb{R}$. The area under the ROC, abbreviated AUC, provides an overall summary of the performance of the classifier, but we are more interested in comparing performance at high levels of sensitivity (high true positive rates) and high levels of specificity (low false positive rates). We therefore make comparisons based on partial AUC (pAUC) measures, as introduced in McClish [16], for the regions of interest. 
The calculation of a specificity-oriented pAUC measure is illustrated in the left panel of Figure 4. In this example, we focus on the area under the curve between 0 and $\mathrm{FP}_{1}$ (call that "Area") and then normalize it to fall between $1 / 2$ and 1 through the transformation

$$
p A U C=\frac{1}{2}\left(1+\frac{\text { Area }-\min }{\max -\min }\right)=\frac{1}{2}\left(1+\frac{\text { Area }-\mathrm{FP}_{1}^{2} / 2}{\mathrm{FP}_{1}-\mathrm{FP}_{1}^{2} / 2}\right) .
$$

Here, max is the area $\mathrm{FP}_{1}$ of the shaded rectangle, and min is the area $\mathrm{FP}_{1}^{2} / 2$ of the triangular portion of the rectangle below the diagonal. An ideal classifier over the interval from 0 to $\mathrm{FP}_{1}$ would have a pAUC of 1 , whereas a random assignment of observations to classes would have a pAUC of $1 / 2$. To focus on high specificity, we consider values of $\mathrm{FP}_{1}$ decreasing from 0.10 to 0, which corresponds to a true negative rate (TNR) increasing from 0.90 to 1 .

The calculation of a sensitivity-oriented pAUC measure on the right side of Figure 4 works similarly. To focus on high sensitivity, we take a rectangle along the top end of the unit square. The lower boundary of that rectangle is defined by a true positive rate (TPR) that we initially set equal to 0.90 and then increase toward 1 . In the normalization of the area for this case, $\min$ is the area to the right of diagonal. We use the $\mathrm{R}$ package $p R O C$ (Robin et al. [18]) to facilitate the calculation and plotting of pAUC values.
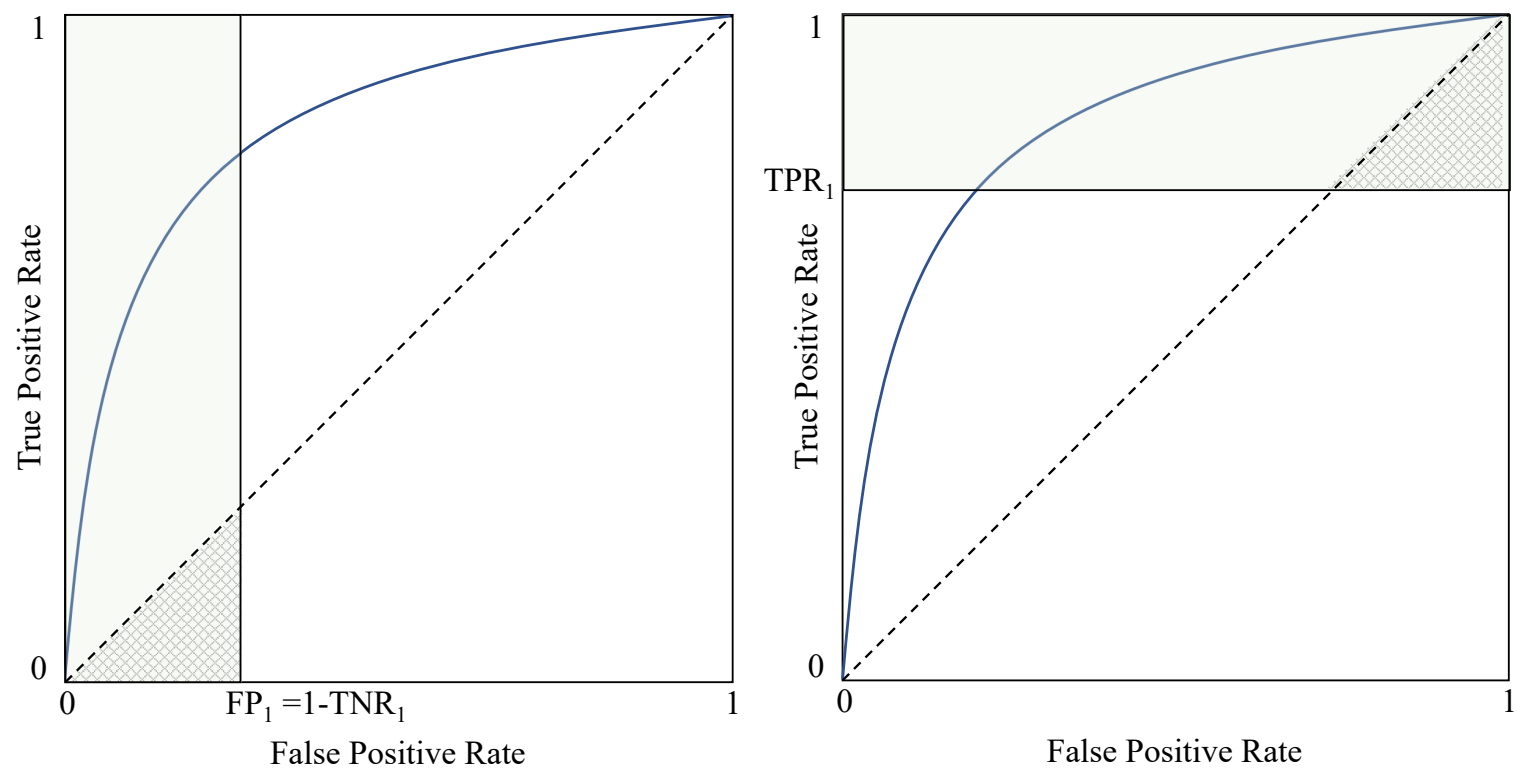

Figure 4: Illustration of a specificity-oriented (left) and sensitivity-oriented (right) pAUC.

Figure 5 compares pAUC values for logistic and exponential classifiers with a sample size of $N=10,000$ for the majority class. Panel (a) plots pAUCs in the high-sensitivity region, with the true positive rate $\mathbb{P}_{1}\left(\beta^{\top} X>t\right)$ increasing from 0.90 toward 1. Among the exponential classifiers, with see that, at high levels of sensitivity, the classifier with $\lambda=0.9$ outperforms the 
classifier with $\lambda=0.5$, which outperforms the classifier with $\lambda=0.1$. Panel (b) focuses on the region of high specificity, where the true negative rate $\mathbb{P}_{0}\left(\beta^{\top} X \leq t\right)$ increases from 0.90 toward 1. Here we see the ordering of the exponential classifiers reversed. This pattern is consistent with our interpretation of the exponential objective in Section 5.4: higher $\lambda$ puts more weight on sensitivity, and lower $\lambda$ puts more weight on specificity.

We see this pattern as the key consideration in choosing $\lambda$. In applications such as disease testing or screening for default risk, where a false negative may be much more costly than a false positive, a larger $\lambda$ should be preferred; but if the goal is to maintain high specificity while optimizing for sensitivity, then a smaller $\lambda$ is more appropriate.

At both extremes, Figure 5 indicates that the performance of the logistic classifier falls between the exponential classifiers with $\lambda=0.1$ and $\lambda=0.5$. We investigate this pattern further in Figure 6, where we consider the effect of a smaller $(N=1,000)$ or larger $(N=50,000)$ sample size. Comparing these results with those in Figure 5 reveals a consistent pattern: as $N$ increases, the performance of the logistic classifier becomes indistinguishable from that of an exponential classifier with small $\lambda$. This pattern is consistent with our theoretical results, Theorems 4.2 4.4 if we think of $\beta_{*}(\lambda)$ as a function of the exponent $\lambda$, then a comparison of (12) and (13) suggests that $\lim _{\lambda \downarrow 0} \beta_{*}(\lambda)=\beta_{*}$, where $\beta_{*}$ is the limiting coefficient vector for ordinary logistic regression.

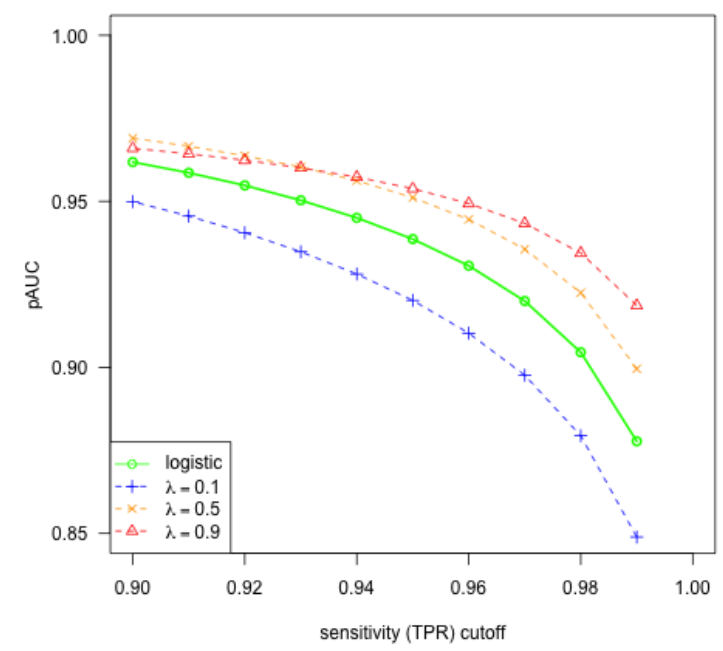

(a) High-sensitivity region

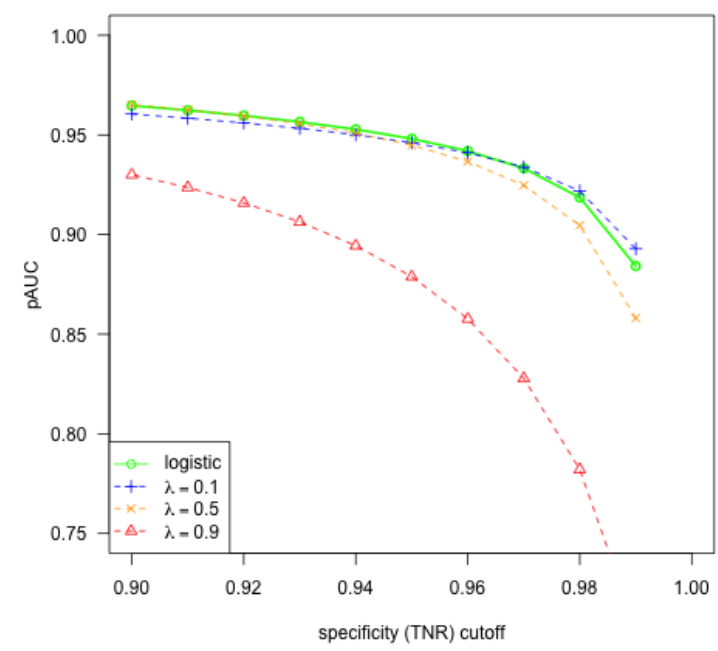

(b) High-specificity region

Figure 5: Comparison of pAUC values for logistic and exponential $(\lambda=0.1,0.5,0.9)$ classifiers 


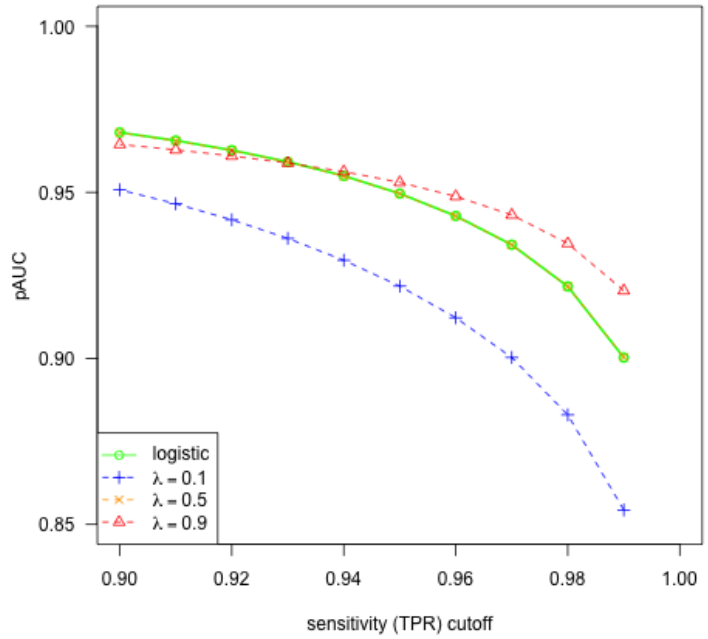

(a) High-sensitivity region, $N=1,000$

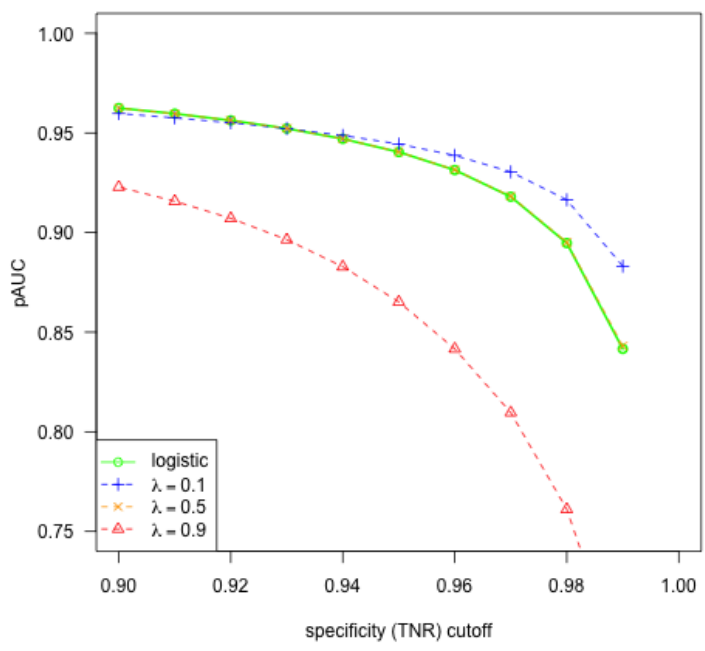

(c) High-specificity region, $N=1,000$

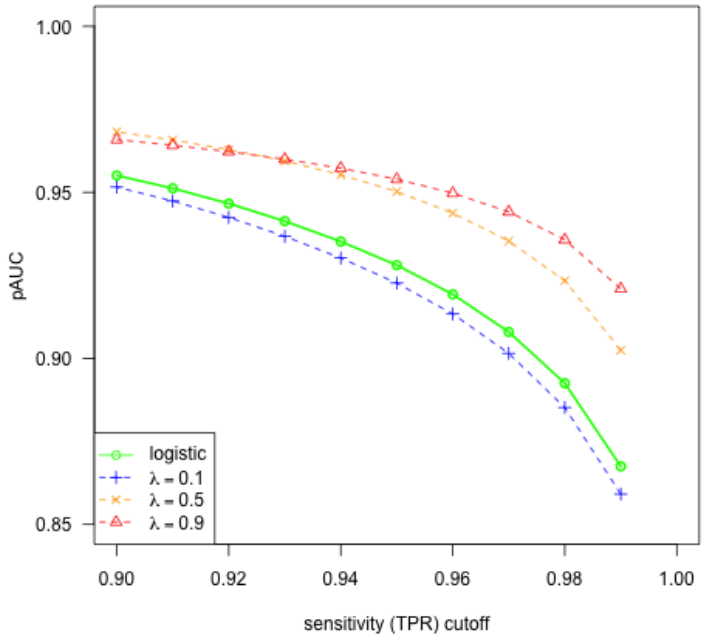

(b) High-sensitivity region, $N=50,000$

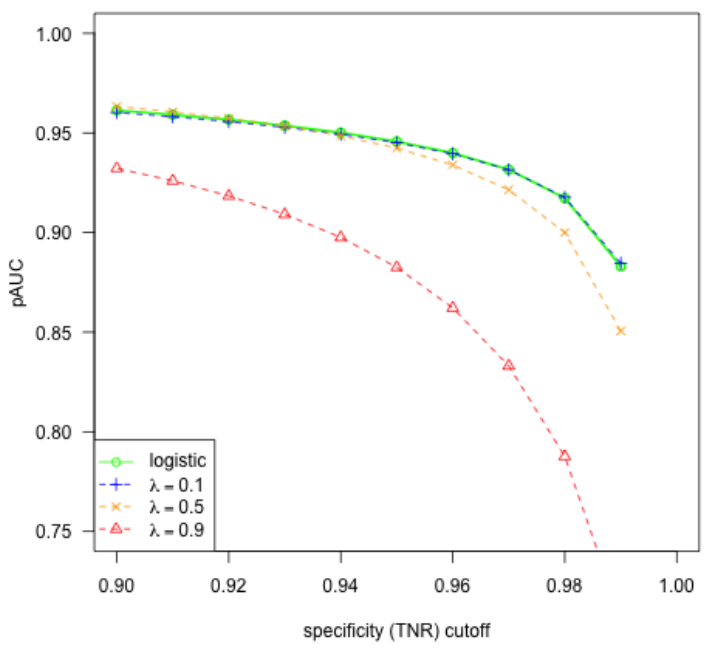

(d) High-specificity region, $N=50,000$

Figure 6: As $N$ increases, the logistic pAUC values move closer to the exponential pAUC values with small $\lambda$ in both the high-sensitivity and high-specificity regions 


\section{A Credit Risk Application}

\subsection{Freddie Mac Data}

The task of classifying borrowers by their credit risk is challenged by imbalanced data in settings where defaults are rare. In this section, we apply ideas from previous sections to quarterly data from the Freddie Mac Single Family Loan-Level Dataset, from 2003 to 2016. The dataset can be accessed from http://www.freddiemac.com/research/datasets/sf_loanlevel_dataset.page. The dataset covers mortgages purchased or guaranteed by Freddie Mac.

Our outcome of interest - the binary label we attach to each loan - is whether the loan defaults within two years of origination. We define a loan to be in default if it is 180 days or more past due. Our goal is to predict this outcome based on loan and borrower features available at origination. This setup is consistent with Li et al. [14, although our sample is much larger.

Figure $7 \mathrm{a}$ plots the number of loans originated in each quarter, and Figure $7 \mathrm{~b}$ plots the default rate over time. We exclude from our analysis all loans that were repurchased within two years of origination. The default rate is almost always less than $0.03 \%$, except around the financial crisis of 2008 when it climbs near 3.5\%. We are thus dealing with extremely imbalanced data and considerable variation in the degree of imbalance.

In predicting outcomes, we use a combination of numerical and categorical attributes. The numerical variables are credit score, original debt-to-income ratio, log of original unpaid principal balance, original loan-to-value ratio, and original interest rate; the categorical variables are number of borrowers (one or more than one), first time homebuyer flag, number of units, occupancy status, loan origination channel, prepayment penalty mortgage flag, property type, and loan purpose. Precise definitions of these variables can be found in the Freddie Mac [8] user guide.

We estimate linear classifiers over a rolling window, for $t=2003, \ldots, 2013$. For $t=2003$, the process works as follows. We use $80 \%$ of loans originated in any of the four quarters of 2003 and their default status in the corresponding quarter of $2005(t+2)$ to estimate a model, reserving the other $20 \%$ of the data for later validation. This is our training data for $t=2003$. We then apply the trained model to the attributes of loans originated in the first quarter of 2004 to predict default status as of the first quarter of $2006(t+3)$. This is our test data for $t=2003$. We apply the same process, retraining the model with $t=2004$, to predict default status in the first quarter of 2007 for loans originated in the first quarter of 2005. Our last forecast is for defaults in the first quarter of 2016, for loans originated in the first quarter of 2014 , trained based on loans originated in $t=2013$. 
(a) Number of Loans

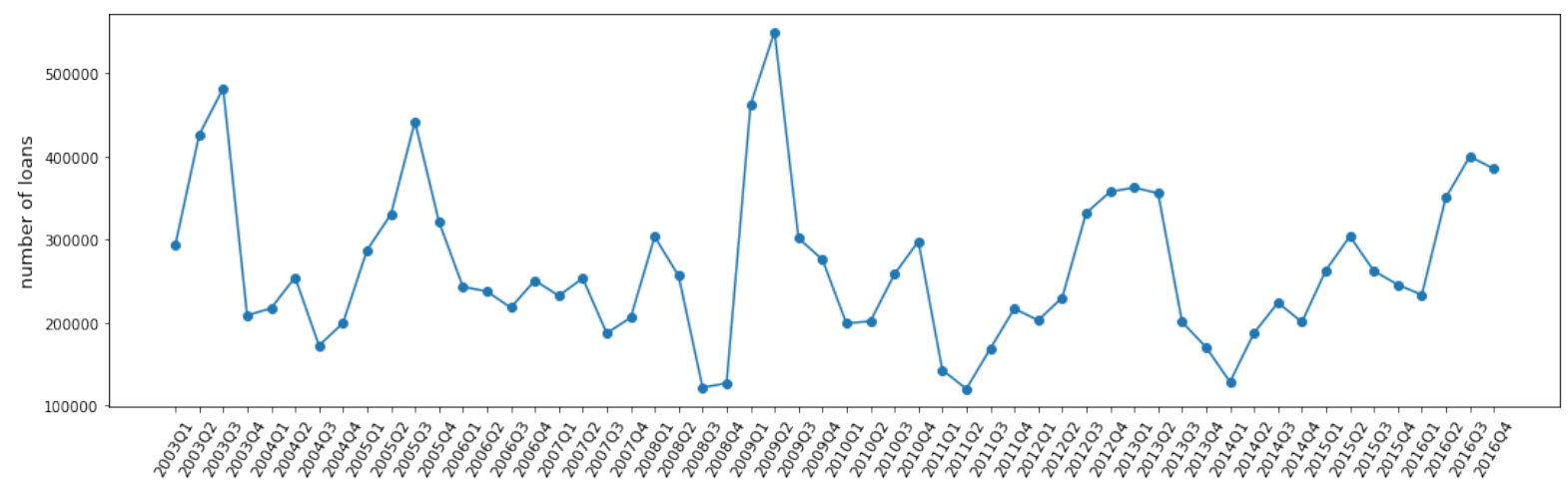

(b) Default Rate over Time

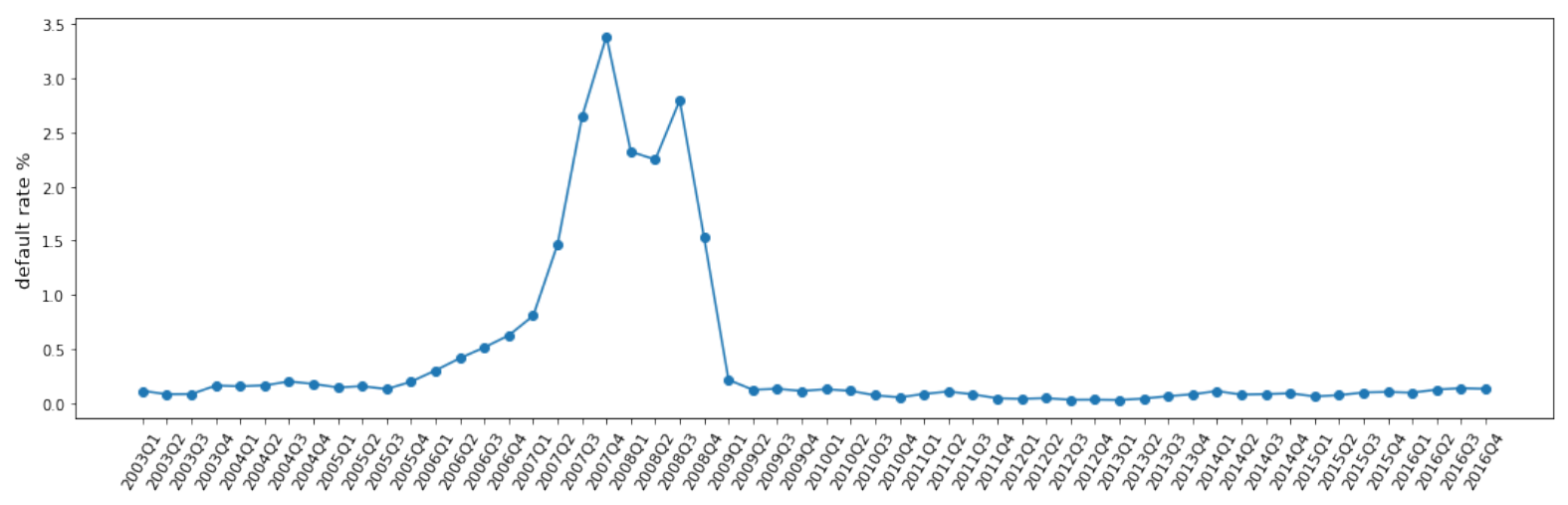

Figure 7: Freddie Mac Summary Data

We remove loans that are missing values for any numerical variables. For each categorical variable, we interpret missing values as a separate category. At each $t$, we check each variable to ensure that at least two distinct values are taken for both majority and minority groups to avoid degeneracy. We omit the variable for that $t$ if the variable fails this check, which happens in fewer than $1 \%$ of cases.

Using this process, we estimate four classifiers at each $t$, using logistic regression and exponential objectives with $\lambda=0.1,0.5,0.9$. In Appendix C.1, we report AUCs for training, validation, and test data for each classifier, for each $t$. All AUCs are above 0.8, indicating that linear classifiers perform reasonably well in this task. The validation AUCs and testing AUCs are all very close to the training AUCs, allaying any concerns about overfitting.

\subsection{High-Sensitivity Classifiers}

We consider a lender that would like to apply a simple first-pass classifier that correctly identifies at least $99 \%$ of customers who would default as high risk. Those classified as high risk would 
then undergo a costlier in-depth review. The lender thus wants the first-pass classifier to have a high TPR to make it highly sensitive to likely defaulters. We have seen that, in highly imbalanced settings, logistic regression becomes similar to an exponential classifier with $\lambda$ close to zero; but we have also seen that in the high-sensitivity region we should prefer to take $\lambda$ close to one. We investigate this comparison using the Freddie Mac data.

For each classifier and each year $t$, we set a classification threshold to achieve a TPR of $99 \%$ in the training data. We then evaluate the TPR and TNR in the test set for each classifier and each year.

Appendix C.2 reports the test TPRs for all methods and all years. In all cases, the test TPR is close to $99 \%$, indicating that the threshold set in the training data works well in the test data. However, we see clear differences in the test TNRs reported in Table 2. In every case, the exponential classifier with $\lambda=0.9$ achieves the best performance, as we expected in this high-sensitivity region. The classifier with $\lambda=0.5$ consistently outperforms the logistic classifier and the case $\lambda=0.1$, and the last two are difficult to distinguish. All of these findings are consistent with our interpretation of the effect of the parameter $\lambda$ and the relationship between the logistic and exponential objectives.

\begin{tabular}{rrrrr}
\hline Year & Logistic & $\lambda=0.1$ & $\lambda=0.5$ & $\lambda=0.9$ \\
\hline 2003 & 28.46 & 28.70 & 33.69 & $\mathbf{3 7 . 9 0}$ \\
2004 & 24.32 & 22.28 & 24.60 & $\mathbf{2 6 . 0 8}$ \\
2005 & 23.73 & 23.91 & 24.76 & $\mathbf{2 6 . 3 4}$ \\
2006 & 22.63 & 23.00 & 26.33 & $\mathbf{2 7 . 9 8}$ \\
2007 & 21.90 & 21.92 & 22.92 & $\mathbf{2 4 . 4 7}$ \\
2008 & 22.35 & 23.57 & 24.57 & $\mathbf{2 5 . 4 5}$ \\
2009 & 27.84 & 25.86 & 29.87 & $\mathbf{3 0 . 8 9}$ \\
2010 & 17.96 & 21.09 & 23.12 & $\mathbf{2 5 . 5 3}$ \\
2011 & 31.17 & 32.29 & 35.82 & $\mathbf{4 1 . 4 9}$ \\
2012 & 33.08 & 32.97 & 34.79 & $\mathbf{4 0 . 9 0}$ \\
2013 & 24.95 & 25.56 & 27.05 & $\mathbf{2 8 . 8 1}$ \\
\hline
\end{tabular}

Table 2: True negative rates (in percent) in test data for classifiers trained at a true positive rate of $99 \%$

\section{3 pAUC plots}

To gain further insight into the comparison of the classifiers, we examine pAUC plots like those introduced in Section 6, but now using the Freddie Mac data. Figure 8 shows results for 2007, but we find the same pattern in all years: as expected, a higher $\lambda$ gives better results in the high-sensitivity region, and a lower $\lambda$ works better in the high-specificity region. The performance of logistic regression is similar to that of $\lambda=0.1$ in the first case but closer to that 
of $\lambda=0.5$ in the second case.

To gauge the statistical significance of these differences, Figure 9 includes $90 \%$ bootstrap confidence intervals around the pAUC curves. For clarity, we compare just two cases, $\lambda=0.9$ in red and $\lambda=0.1$ in blue. The confidence bands barely overlap, indicating that the ordering of the two curves is reliable.

\section{Concluding Remarks}

We have shown that a broad family of linear discriminant functions have explicit limits as the sample size of one class grows while the sample size for the other remains fixed. Linear discriminant functions defined by bounded or asymptotically polynomial weight functions share a common limit with logistic regression. A wider range of limits apply using asymptotically exponential weights. The limits of these classifiers reflect different types of robustness or conservatism towards worst-case false-positive and false-negative errors. We illustrated these ideas through numerical examples and an application to credit risk in predicting mortgage defaults.

Our analysis is limited to linear discriminant functions. Linear classifiers are widely used in practice, at least in part because they are easy to interpret. Note, also, that the features used for classification could include scores computed from nonlinear models. In other words, a linear discriminant function can be used to aggregate results from multiple models. It would be interesting to know if similar limits hold for scoring rules derived from regression trees or neural networks. The loss functions used to optimize these types of rules over parameters are typically nonconvex, which significantly complicates any analysis of their limiting behavior.

\section{References}

[1] Brown, L.D. (1986) Fundamental of Statistical Exponential Families, Institute of Mathematical Statistics, Hayward, California.

[2] Chawla, N.V., Bowyer, K.W., Hall, L.O., and Kegelmeyer, W.P. (2002) SMOTE: Synthetic Minority Over-Sampling Technique. Journal of Artificial Intelligence Research 16:321-357.

[3] Csiszar, I. (1975) I-Divergence Geometry of Probability Distributions and Minimization Problems. Annals of Probability 3(1):146-158.

[4] Drummond, C. and Holte, R.C. (2003) C4. 5, Class Imbalance, and Cost Censitivity: Why Under-Sampling Beats Over-Sampling. Workshop on Learning from Imbalanced Datasets, Washington, D.C. 


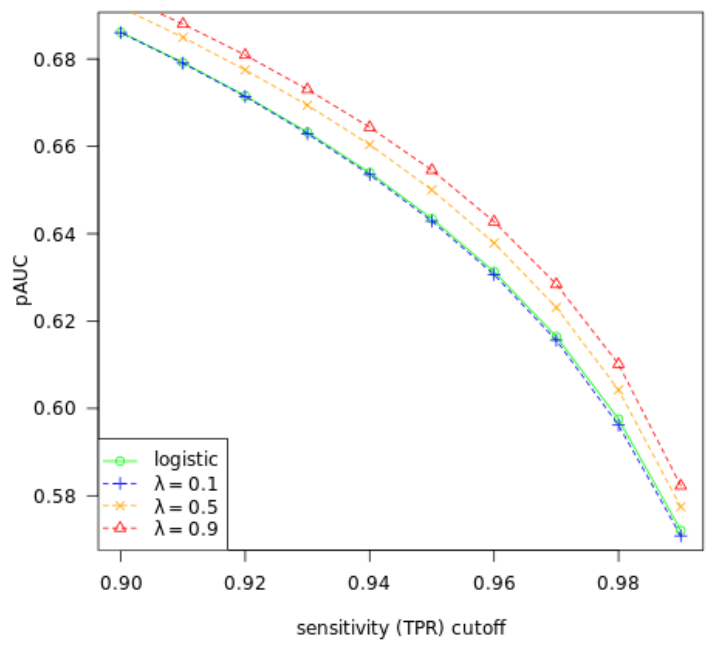

(a) High-sensitivity region

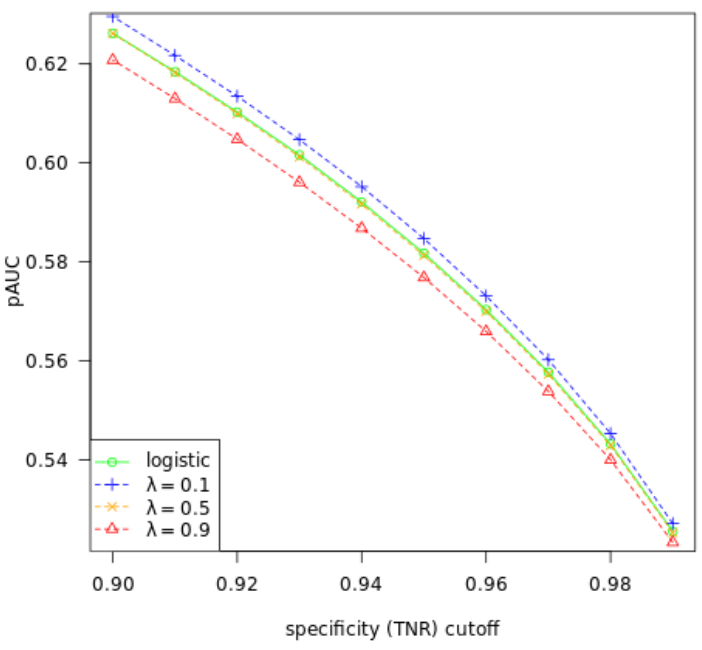

(b) High-specificity region

Figure 8: Comparison pAUC values in training data for logistic and exponential $(\lambda=$ 0.1, 0.5, 0.9) classifiers using Freddie Mac loan data
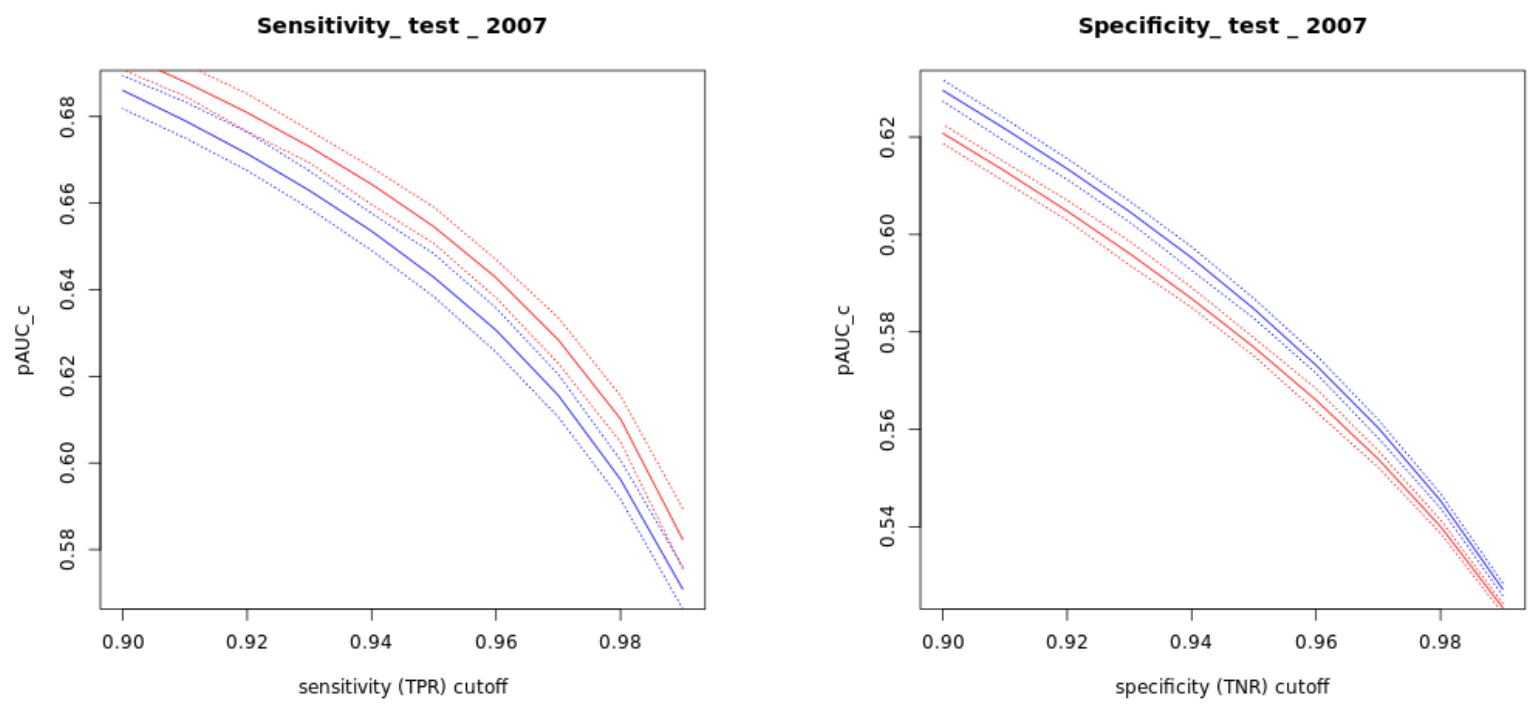

Figure 9: Training pAUC plots with $90 \%$ confidence bands for $\lambda=0.1$ (blue) and $\lambda=0.9$ (red). 
[5] Eguchi, S., and Copas, J. (2002) A Class of Logistic-Type Discriminant Functions. Biometrika 89(1):1-22.

[6] van Erven, T., and Harremoës, P. (2014) Rényi Divergence and Kullback-Leibler Divergence, IEEE Transactions on Information Theory 60(7):3793-3820.

[7] Friedman, J., Hastie, T., and Tibshirani, R. (2000) Additive Logistic Regression: A Statistical View of Boosting. Annals of Statistics 28(2):337-407.

[8] Freddie Mac (2021) Single Family Loan-Level Dataset General User Guide. Freddie Mac, McLean, Virginia.

[9] Freund, Y., and Schapire, R.E. (1997) A Decision-Theoretic Generalization of On-Line Learning and an Application to Boosting. Journal of Computer and System Sciences 55:119139.

[10] Gill, P.M., Pearce, C.E.M., and Pečarić, J. (1997) Hadamard's Inequality for r-Convex Functions, Journal of Mathematical Analysis and Applications 215:461-470.

[11] Kubat, M., and Matwin, S. (1997) Addressing the Curse of Imbalanced Training Sets: One-Sided Selection. Proceedings of the 14th International Conference in Machine Learning. Morgan Kaufmann, San Francisco, 179-186.

[12] Kullback, S., and Leibler, R.A. (1951) On Information and Sufficiency. Annals of Mathematical Statistics 22(1):79-86.

[13] Lehmann, E.L., and Romano, J.P. (2005) Testing Statistical Hypotheses, 3rd Ed., Springer.

[14] Li, Y., Bellotti, T., and Adams, N. (2019) Issues Using Logistic Regression with Class Imbalance, with a Case Study from Credit Risk Modelling. Foundations of Data Science $1(4): 389-417$.

[15] Liu, X.-Y., Wu, J., and Zhou, Z.-H. (2008) Exploratory Undersampling for Class-Imbalance Learning. IEEE Transactions on Systems, Man, and Cybernetics, Part B (Cybernetics) $39(2): 539-550$.

[16] McClish, D. (1989) Analyzing a Portion of the ROC Curve. Medical Decision Making 9:190-195.

[17] Owen, A. (2007) Infinitely Imbalanced Logistic Regression. Journal of Machine Learning Research 8:761-773. 
[18] Robin, X., Turck, N., Hainard, A., Tiberti, N., Lisacek, F., Sanchez, J.-C, and Müller, M. (2011) pROC: an Open-source Package for R and S+ to Analyze and Compare ROC Curves. BMC Bioinformatics, 12:77.

[19] Silvapulle, M. (1981) On the Existence of Maximum Likelihood Estimates for the Binomial Response Models. Journal of the Royal Statistical Society, Series B 43:310-313.

\section{A Proofs for Section 3}

Proof of Lemma 3.1. Differentiating (4) yields

$$
\frac{d U}{d u}=w(u), \quad \frac{d^{2} U}{d u^{2}}=w^{\prime}(u)
$$

and

$$
\frac{d V}{d u}=e^{u} w(u), \quad \frac{d^{2} V}{d u^{2}}=e^{u}\left(w(u)+w^{\prime}(u)\right),
$$

so Condition 2 implies that $U$ is concave and $V$ is strictly convex. It follows that each term $-U\left(\alpha+\beta^{\top} x_{i}\right)$ and $V\left(\alpha+\beta^{\top} x\right)$ is convex in $(\alpha, \beta)$, and thus that $\bar{C}$ in $(7)$ is convex.

To establish strict convexity of $\bar{C}$, consider any $\left(\alpha_{1}, \beta_{1}\right),\left(\alpha_{2}, \beta_{2}\right)$ where $\bar{C}$ is finite and any $\nu \in(0,1)$. Let $A=\left\{x \in \mathbb{R}^{d}: \alpha_{1}+\beta_{1}^{\top} x=\alpha_{2}+\beta_{2}^{\top} x\right\}$, and let $\bar{A}$ be its complement in $\mathbb{R}^{d}$. Then

$$
\begin{aligned}
\int & V\left(\nu\left(\alpha_{1}+\beta_{1}^{\top} x\right)+(1-\nu)\left(\alpha_{2}+\beta_{2}^{\top} x\right)\right) d F_{0}(x) \\
= & \int_{\bar{A}} V\left(\nu\left(\alpha_{1}+\beta_{1}^{\top} x\right)+(1-\nu)\left(\alpha_{2}+\beta_{2}^{\top} x\right)\right) d F_{0}(x)+\int_{A} V\left(\nu\left(\alpha_{1}+\beta_{1}^{\top} x\right)+(1-\nu)\left(\alpha_{2}+\beta_{2}^{\top} x\right)\right) d F_{0}(x) \\
< & \nu \int_{\bar{A}} V\left(\alpha_{1}+\beta_{1}^{\top} x\right) d F_{0}(x)+(1-\nu) \int_{\bar{A}} V\left(\alpha_{2}+\beta_{2}^{\top} x\right) d F_{0}(x) \\
& +\int_{A} V\left(\nu\left(\alpha_{1}+\beta_{1}^{\top} x\right)+(1-\nu)\left(\alpha_{2}+\beta_{2}^{\top} x\right)\right) d F_{0}(x) \\
\leq & \nu \int V\left(\alpha_{1}+\beta_{1}^{\top} x\right) d F_{0}(x)+(1-\nu) \int V\left(\alpha_{2}+\beta_{2}^{\top} x\right) d F_{0}(x) .
\end{aligned}
$$

The strict inequality follows from the strict convextiy of $V$ and the requirement that the support of $F_{0}$ not be contained within $A$. Strict convexity of $\bar{C}$ follows.

Lemma A.1 (Partials). Suppose Conditions 1, 2, and 4 hold. Then for any $(\alpha, \beta) \in \Theta$,

$$
\begin{gathered}
\frac{\partial \bar{V}}{\partial \alpha}(\alpha, \beta)=\int e^{\alpha+\beta^{\top} x} w\left(\alpha+\beta^{\top} x\right) d F_{0}(x) \\
\frac{\partial \bar{V}}{\partial \beta}(\alpha, \beta)=\int x e^{\alpha+\beta^{\top} x} w\left(\alpha+\beta^{\top} x\right) d F_{0}(x),
\end{gathered}
$$

where $\partial \bar{V} / \partial \beta$ denotes the gradient with respect to $\beta$. 
Proof. If $(\alpha, \beta) \in \Theta$, then for any $\theta \in \mathbb{R}^{d+1}$, we can find $\epsilon>0$ such that $(\alpha, \beta) \pm \epsilon \theta \in \Theta$. Let $V_{\epsilon}(x)=V\left(\alpha_{\epsilon}+x^{\top} \beta_{\epsilon}\right)$, where $\left(\alpha_{\epsilon}, \beta_{\epsilon}\right)=(\alpha, \beta)+\epsilon \theta$. Then $V_{\epsilon}(x)$ is convex in $\epsilon$ for all $x$. For $0<h<\epsilon$, convexity implies

$$
\frac{V_{0}(x)-V_{-\epsilon}(x)}{\epsilon} \leq \frac{V_{h}(x)-V_{0}(x)}{h} \leq \frac{V_{\epsilon}(x)-V_{0}(x)}{\epsilon},
$$

so

$$
\left|\frac{V_{0}(x)-V_{h}(x)}{h}\right| \leq \frac{V_{-\epsilon}(x)+V_{0}(x)+V_{\epsilon}(x)}{\epsilon} .
$$

The right side is integrable with respect to $F_{0}$, so the dominated convergence theorem justifies the exchange of limit and expectation; i.e.,

$$
\left.\partial_{\epsilon} \bar{V}((\alpha, \beta)+\epsilon \theta)\right|_{\epsilon=0}=\left.\int \partial_{\epsilon} V_{\epsilon}(x)\right|_{\epsilon=0} d F_{0}(x) .
$$

Choosing $\theta$ to be a vector of 0 s with a single 1 yields the partial derivatives in the statement of the lemma.

Proof of Lemma 3.2. If $F_{0}$ surrounds a point, its support cannot be contained in a lowerdimensional subspace, so we know from Lemma 3.1 that $\bar{C}$ is strictly convex in $(\alpha, \beta)$. Strict convexity implies that either $\bar{C}$ has a unique minimizer or it approaches $-\infty$ along some ray $\left\{\left(\lambda \alpha_{0}, \lambda \beta_{0}\right) \mid 0 \leq \lambda<\infty\right\}$, with $\left(\alpha_{0}, \beta_{0}\right) \in \Theta$ not identically zero. We will show that the latter case is not possible.

First consider the case that for some finite $\bar{\lambda}>0,\left(\bar{\lambda} \alpha_{0}, \bar{\lambda} \beta_{0}\right)$ is a boundary point of $\Theta$ and therefore not in the open set $\Theta$. Then lower semicontinuity of $\bar{V}$ implies that

$$
\liminf _{\lambda \uparrow \bar{\lambda}} \bar{V}\left(\lambda \alpha_{0}, \lambda \beta_{0}\right) \geq \bar{V}\left(\bar{\lambda} \alpha_{0}, \bar{\lambda} \beta_{0}\right)=\infty
$$

As $U\left(\alpha+\beta^{\top} x\right)$ is finite for all finite $(\alpha, \beta)$ and $x, \bar{C}\left(\lambda \alpha_{0}, \lambda \beta_{0}\right) \rightarrow \infty$ as $\lambda \uparrow \bar{\lambda}$. It therefore suffices to consider the case that $\left(\lambda \alpha_{0}, \lambda \beta_{0}\right) \in \Theta$ for all $\lambda \geq 0$. We treat separately the cases $\beta_{0}=0\left(\right.$ with $\left.\alpha_{0} \neq 0\right)$ and $\beta_{0}^{\top} \beta_{0}=1$. The normalization in the second case is justified by our scaling by $\lambda$.

Case 1: $\beta_{0}=0, \alpha_{0} \neq 0$. Using Lemma A.1 to differentiate $\bar{V}$, we get

$$
\frac{\partial \bar{C}\left(\lambda \alpha_{0}, \lambda \beta_{0}\right)}{\partial \lambda}=\left(-n+N e^{\lambda \alpha_{0}}\right) w\left(\lambda \alpha_{0}\right) \alpha_{0}
$$

If $\alpha_{0}>0$, then for $\lambda$ large, $e^{\lambda \alpha_{0}}>n / N$, and the derivative is strictly positive. If $\alpha_{0}<0$, then for $\lambda$ large, $e^{\lambda \alpha_{0}}<n / N$ and the derivative is again strictly positive. 
Case 2: $\beta_{0}^{\top} \beta_{0}=1$. Again using Lemma A.1 to differentiate $\bar{V}$, we get

$$
\begin{aligned}
\frac{\partial \bar{C}\left(\lambda \alpha_{0}, \lambda \beta_{0}\right)}{\partial \lambda}= & \sum_{i=1}^{n}-w\left(\lambda \alpha_{0}+\lambda \beta_{0}^{\top} x_{i}\right)\left(\alpha_{0}+\beta_{0}^{\top} x_{i}\right) \\
& +N \int e^{\lambda \alpha_{0}+\lambda \beta_{0}^{\top} x} w\left(\lambda \alpha_{0}+\lambda \beta_{0}^{\top} x\right)\left(\alpha_{0}+\beta_{0}^{\top} x\right) d F_{0}(x) \\
= & \sum_{i: \alpha_{0}+\beta_{0}^{\top} x_{i}<0}-w\left(\lambda \alpha_{0}+\lambda \beta_{0}^{\top} x_{i}\right)\left(\alpha_{0}+\beta_{0}^{\top} x_{i}\right) \\
& +\sum_{i: \alpha_{0}+\beta_{0}^{\top} x_{i}>0}-w\left(\lambda \alpha_{0}+\lambda \beta_{0}^{\top} x_{i}\right)\left(\alpha_{0}+\beta_{0}^{\top} x_{i}\right) \\
& +N \int_{\alpha_{0}+\beta_{0}^{\top} x<0} e^{\lambda \alpha_{0}+\lambda \beta_{0}^{\top} x} w\left(\lambda \alpha_{0}+\lambda \beta_{0}^{\top} x\right)\left(\alpha_{0}+\beta_{0}^{\top} x\right) d F_{0}(x) \\
& +N \int_{\alpha_{0}+\beta_{0}^{\top} x>0} e^{\lambda \alpha_{0}+\lambda \beta_{0}^{\top} x} w\left(\lambda \alpha_{0}+\lambda \beta_{0}^{\top} x\right)\left(\alpha_{0}+\beta_{0}^{\top} x\right) d F_{0}(x) .
\end{aligned}
$$

We will show that as $\lambda$ increases, the liminf of the derivative on the left is strictly positive. We will prove this by showing that (29) and (30) approach zero as $\lambda$ increases, and the sum of (28) and (31) remains positive and bounded away from zero as $\lambda$ increases.

For $29, \alpha_{0}+\beta_{0}^{\top} x_{i}>0$ implies $\lambda\left(\alpha_{0}+\beta_{0}^{\top} x_{i}\right) \rightarrow \infty$, and thus $w\left(\lambda \alpha_{0}+\lambda \beta_{0}^{\top} x_{i}\right) \rightarrow 0$.

To evaluate the limit of $(30)$, we use the fact that $e^{s} w(s)$ is increasing to write

$$
\int_{\alpha_{0}+\beta_{0}^{\top} x<0}\left|e^{\lambda \alpha_{0}+\lambda \beta_{0}^{\top} x} w\left(\lambda \alpha_{0}+\lambda \beta_{0}^{\top} x\right)\left(\alpha_{0}+\beta_{0}^{\top} x\right)\right| d F_{0}(x) \leq w(0) \int_{\alpha_{0}+\beta_{0}^{\top} x<0}\left|\alpha_{0}+\beta_{0}^{\top} x\right| d F_{0}(x)<\infty,
$$

as $F_{0}$ has finite mean. The limit of the integral (30) is therefore the integral of the limit

$$
\lim _{\lambda \rightarrow \infty} e^{\lambda \alpha_{0}+\lambda \beta_{0}^{\top} x} w\left(\lambda \alpha_{0}+\lambda \beta_{0}^{\top} x\right)\left(\alpha_{0}+\beta_{0}^{\top} x\right)=0,
$$

for all $\alpha_{0}+\beta_{0}^{\top} x<0$, because $e^{s} w(s) \rightarrow 0$ as $s \rightarrow-\infty$.

The terms in (28) and (31) are nonnegative. We need to show that at least one of them remains bounded away from zero. Using the fact that $w(s)>0$ is decreasing we get a lower bound for (28),

$$
\begin{aligned}
\sum_{i: \alpha_{0}+\beta_{0}^{\top} x_{i}<0}-w\left(\lambda \alpha_{0}+\lambda \beta_{0}^{\top} x_{i}\right)\left(\alpha_{0}+\beta_{0}^{\top} x_{i}\right) & \geq-w(0) \sum_{i: \alpha_{0}+\beta_{0}^{\top} x_{i}<0}\left(\alpha_{0}+\beta_{0}^{\top} x_{i}\right) \\
& \geq-w(0) \sum_{i=1}^{n}\left(\alpha_{0}+\beta_{0}^{\top} x_{i}\right) \\
& =-n \cdot w(0)\left(\alpha_{0}+\beta_{0}^{\top} \bar{x}\right) .
\end{aligned}
$$


Using the fact that $e^{s} w(s)$ is increasing we get a lower bound for (31),

$$
\begin{aligned}
\int_{\alpha_{0}+\beta_{0}^{\top} x>0} e^{\lambda \alpha_{0}+\lambda \beta_{0}^{\top} x} w( & \left.\lambda \alpha_{0}+\lambda \beta_{0}^{\top} x\right)\left(\alpha_{0}+\beta_{0}^{\top} x\right) d F_{0}(x) \\
& \geq w(0) \int_{\alpha_{0}+\beta_{0}^{\top} x>0}\left(\alpha_{0}+\beta_{0}^{\top} x\right) d F_{0}(x) \\
& \geq w(0) \epsilon \int_{\alpha_{0}+\beta_{0}^{\top} x>\epsilon} d F_{0}(x) \\
& =w(0) \epsilon \int_{\beta_{0}^{\top}(x-\bar{x})>\epsilon-\left(\alpha_{0}+\beta_{0}^{\top} \bar{x}\right)} d F_{0}(x) .
\end{aligned}
$$

If $\alpha_{0}+\beta_{0}^{\top} \bar{x}<0$, then 32 is strictly positive; if $\alpha_{0}+\beta_{0}^{\top} \bar{x} \geq 0$, then the surrounding condition implies that (33) is strictly positive, for sufficiently small $\epsilon>0$.

\section{B Proofs for Section 4}

\section{B.1 Proof of Lemma 4.1}

We separate the proof into two steps, first showing that $\alpha_{N}+\beta_{N}^{\top} \bar{x} \rightarrow-\infty$, then showing [10. Within each step, we provide separate arguments for different categories of weight functions $w$.

Lemma B.1 (Step 1). Under the condition of Lemma 4.1, $\alpha_{N}+\beta_{N}^{\top} \bar{x} \rightarrow-\infty$.

Proof of Lemma B.1 for Bounded $w$. At any $(\alpha, \beta) \in \Theta$,

$$
\begin{aligned}
\frac{\partial \bar{C}}{\partial \alpha} & =\sum_{i=1}^{n}-w\left(\alpha+\beta^{\top} x_{i}\right)+N \int e^{\alpha+\beta^{\top} x} w\left(\alpha+\beta^{\top} x\right) d F_{0}(x) \\
& =\sum_{i=1}^{n}-w\left(\alpha+\beta^{\top} x_{i}\right)+N \int e^{\alpha+\beta^{\top} \bar{x}+\beta^{\top}(x-\bar{x})} w\left(\alpha+\beta^{\top} \bar{x}+\beta^{\top}(x-\bar{x})\right) d F_{0}(x) \\
& \geq-n C+N e^{\alpha+\beta^{\top} \bar{x}} w\left(\alpha+\beta^{\top} \bar{x}\right) \int_{\beta^{\top}(x-\bar{x}) \geq 0} d F_{0}(x) \\
& \geq-n C+N e^{\alpha+\beta^{\top} \bar{x}} w\left(\alpha+\beta^{\top} \bar{x}\right) \delta
\end{aligned}
$$

where going from second to third line we used the conditions that $w(u)$ is upper bounded by $C$ and $w(u) e^{u}$ is increasing, and the $\delta>0$ in the last line is from the surrounding condition (9).

Take $N>2 n C / \delta$. Consider any $(\alpha, \beta)$ for which $e^{\alpha+\beta^{\top} \bar{x}} w\left(\alpha+\beta^{\top} \bar{x}\right)>2 n C / N \delta$. For any such $(\alpha, \beta)$, (34) implies that $\partial \bar{C} / \partial \alpha>0$. It follows that no such $(\alpha, \beta)$ can be optimal; the optimal $\left(\alpha_{N}, \beta_{N}\right)$ must satisfy the reverse inequality

$$
e^{\alpha_{N}+\beta^{\top} \bar{x}} w\left(\alpha_{N}+\beta^{\top} \bar{x}\right) \leq 2 n C / N \delta
$$

from which we get $\alpha_{N}+\beta_{N}^{\top} \bar{x} \rightarrow-\infty$. 
Proof of Lemma B.1 for Asymptotically Exponential $w$. Because $w(u) \sim C e^{-\lambda u}$ as $u \rightarrow-\infty$, we have $w(u) \leq C_{1} e^{-\lambda u}$, for all $u \leq u_{0}$, for some $u_{0}$ and some $C_{1}>C$. We derive upper and lower bounds for $U$ and $V$, taking $C_{U}=C_{V}=0$ for the constants in (4). For $U(s)$, we have

$$
U(s) \leq 0
$$

for all $s$. For $s \leq u_{0}$, if we set $C_{2}=U\left(u_{0}\right)+\frac{C_{1}}{\lambda} e^{-\lambda u_{0}}$, then $w(u) \leq C_{1} e^{-\lambda u}, u \leq u_{0}$, implies

$$
-\frac{C_{1}}{\lambda} e^{-\lambda s}+C_{2}=-\int_{s}^{u_{0}} C_{1} e^{-\lambda u} d u+U\left(u_{0}\right) \leq-\int_{s}^{u_{0}} w(u) d u+U\left(u_{0}\right)=U(s) .
$$

Recall that $w(u) \geq C_{L} e^{-\lambda_{1} u}$, for all $u$, with $\lambda_{1} \in(0,1)$. It follows that

$$
V(s) \geq \int_{-\infty}^{s} C_{L} e^{\left(1-\lambda_{1}\right) u} d u=\frac{C_{L}}{1-\lambda_{1}} e^{\left(1-\lambda_{1}\right) s},
$$

for all $s$. For $s \leq u_{0}$, we further have

$$
V(s) \leq \int_{-\infty}^{s} C_{1} e^{(1-\lambda) u} d u=\frac{C_{1}}{1-\lambda} e^{(1-\lambda) s} .
$$

Applying the bounds (35) and (37) to the definition of $\bar{C}(\alpha, \beta)$ in (7), we get

$$
\begin{aligned}
\bar{C}(\alpha, \beta) & \geq N \frac{C_{L}}{1-\lambda_{1}} \int e^{\left(1-\lambda_{1}\right)\left(\alpha+\beta^{\top} x\right)} d F_{0}(x) \\
& =N \frac{C_{L}}{1-\lambda_{1}} e^{\left(1-\lambda_{1}\right)\left(\alpha+\beta^{\top} \bar{x}\right)} \int e^{\left(1-\lambda_{1}\right) \beta^{\top}(x-\bar{x})} d F_{0}(x) \\
& \geq N \frac{C_{L}}{1-\lambda_{1}} e^{\left(1-\lambda_{1}\right)\left(\alpha+\beta^{\top} \bar{x}\right)} \int_{\beta^{\top}(x-\bar{x}) \geq 0} e^{\left(1-\lambda_{1}\right) \beta^{\top}(x-\bar{x})} d F_{0}(x) \\
& \geq N \frac{C_{L}}{1-\lambda_{1}} e^{\left(1-\lambda_{1}\right)\left(\alpha+\beta^{\top} \bar{x}\right)} \int_{\beta^{\top}(x-\bar{x}) \geq 0} d F_{0}(x) \\
& \geq N \frac{C_{L}}{1-\lambda_{1}} e^{\left(1-\lambda_{1}\right)\left(\alpha+\beta^{\top} \bar{x}\right)} \delta
\end{aligned}
$$

where $\delta>0$ is the parameter in the surrounding condition (9). In particular, at the minimizer $\left(\alpha_{N}, \beta_{N}\right)$,

$$
\bar{C}\left(\alpha_{N}, \beta_{N}\right) \geq N \frac{C_{L}}{1-\lambda_{1}} e^{\left(1-\lambda_{1}\right)\left(\alpha_{N}+\beta_{N}^{\top} \bar{x}\right)} \delta .
$$

Now, set $\beta=0$. For $\alpha \leq u_{0}$, applying the lower bound in (36) and upper bound in (38) yields

$$
\bar{C}(\alpha, 0) \leq \frac{C_{1}}{\lambda} n e^{-\lambda \alpha}-C_{2}+\frac{N C_{1}}{1-\lambda} e^{(1-\lambda) \alpha},
$$

For $N$ large enough that $-\log N \leq u_{0}$, setting $\alpha=-\log N$ yields

$$
\bar{C}(-\log N, 0) \leq \frac{C_{1}}{\lambda} n N^{\lambda}-n C_{2}+\frac{C_{1}}{1-\lambda} N^{\lambda}=N^{\lambda}\left(\frac{n C_{1}}{\lambda}+\frac{C_{1}}{1-\lambda}\right)-n C_{2} .
$$


Optimality of $\left(\alpha_{N}, \beta_{N}\right)$ therefore implies

$$
\bar{C}\left(\alpha_{N}, \beta_{N}\right) \leq \bar{C}(-\log N, 0) \leq N^{\lambda}\left(\frac{n C_{1}}{\lambda}+\frac{C_{1}}{1-\lambda}\right)-n C_{2} .
$$

Combining 39 and 40, , we get

$$
\frac{C_{L}}{1-\lambda_{1}} e^{\left(1-\lambda_{1}\right)\left(\alpha_{N}+\beta_{N}^{\top} \bar{x}\right)} \delta \leq N^{\lambda-1}\left(\frac{n C_{1}}{\lambda}+\frac{C_{1}}{1-\lambda}\right)-\frac{n C_{2}}{N} \rightarrow 0,
$$

which implies $\alpha_{N}+\beta_{N}^{\top} \bar{x} \rightarrow-\infty$, as claimed.

Proof of Lemma B.1 for Asymptotically Polynomial w. We will first show that

$$
\min _{i} \alpha_{N}+\beta_{N}^{\top} x_{i} \rightarrow-\infty
$$

Arguing exactly as in (34) but with $\bar{x}$ replaced by $x_{j}$, for any $j$ and any $(\alpha, \beta)$, we get

$$
\begin{aligned}
\frac{\partial \bar{C}}{\partial \alpha}(\alpha, \beta) & \geq \sum_{i=1}^{n}-w\left(\alpha+\beta^{\top} x_{i}\right)+N e^{\alpha+\beta^{\top} x_{j}} w\left(\alpha+\beta^{\top} x_{j}\right) \delta \\
& \geq-n \max _{i} w\left(\alpha+\beta^{\top} x_{i}\right)+N e^{\alpha+\beta^{\top} x_{j}} w\left(\alpha+\beta^{\top} x_{j}\right) \delta,
\end{aligned}
$$

where $\delta>0$ is the parameter in the surrounding condition (9), which we may take to be the same for all $j=1, \ldots, n$. Let $j(N) \in \operatorname{argmin}_{i}\left\{\alpha_{N}+\beta_{N}^{\top} x_{i}\right\}$. Because $-w(s)$ and $e^{s} w(s)$ are monotonically increasing, at the minimizer $\left(\alpha_{N}, \beta_{N}\right)$ we have

$$
\begin{aligned}
0=\frac{\partial \bar{C}}{\partial \alpha}\left(\alpha_{N}, \beta_{N}\right) & \geq-n w\left(\alpha_{N}+\beta_{N}^{\top} x_{j(N)}\right)+N e^{\alpha_{N}+\beta_{N}^{\top} x_{j(N)}} w\left(\alpha_{N}+\beta_{N}^{\top} x_{j(N)}\right) \delta \\
& =w\left(\alpha_{N}+\beta_{N}^{\top} x_{j(N)}\right)\left(-n+N e^{\alpha_{N}+\beta_{N}^{\top} x_{j(N)}} \delta\right),
\end{aligned}
$$

so, $\alpha_{N}+\beta_{N}^{\top} x_{j(N)} \rightarrow-\infty$, which is 41 .

We now use 41) to show that $\alpha_{N}+\beta_{N}^{\top} \bar{x} \rightarrow-\infty$. For $j=1, \ldots, n$, we introduce the centered loss (centered around $x_{j}$ )

$$
C^{j}(\alpha, \beta)=\sum_{i=1}^{n}-U\left(\alpha+\beta^{\top}\left(x_{i}-x_{j}\right)\right)+N \int V\left(\alpha+\beta^{\top}\left(x-x_{j}\right)\right) d F_{0}(x) .
$$

With $\left(\alpha_{N}, \beta_{N}\right)$ the minimizer of $\bar{C}$, the centered loss $C^{j}$ is minimized at $\left(\alpha_{N}^{j}, \beta_{N}^{j}\right)$, where

$$
\alpha_{N}^{j}=\alpha_{N}+\beta_{N}^{\top} x_{j}, \quad \beta_{N}^{j}=\beta_{N}
$$

Consider

$$
\begin{aligned}
& C^{j}(\alpha, 0)-C^{j}(\alpha, \beta) \\
& =\sum_{i=1}^{n}\left[-U(\alpha)+U\left(\alpha+\beta^{\top}\left(x_{i}-x_{j}\right)\right)\right]+N V(\alpha)-N \int V\left(\alpha+\beta^{\top}\left(x-x_{j}\right)\right) d F_{0}(x) .
\end{aligned}
$$


Since $U$ is concave with $d U / d u=w(u)$,

$$
U\left(\alpha+\beta^{\top}\left(x_{i}-x_{j}\right)\right) \leq U(\alpha)+w(\alpha) \beta^{\top}\left(x_{i}-x_{j}\right)
$$

and

$$
\sum_{i=1}^{n}\left[-U(\alpha)+U\left(\alpha+\beta^{\top}\left(x_{i}-x_{j}\right)\right)\right] \leq \sum_{i=1}^{n} w(\alpha) \beta^{\top}\left(x_{i}-x_{j}\right) \leq n w(\alpha)\|\beta\| C,
$$

where $C=\max _{i, j}\left\|x_{i}-x_{j}\right\|$.

Similarly, $V$ is strictly convex and strictly positive with $d V / d u=e^{u} w(u)$, so, for $x \neq x_{j}$,

$$
V\left(\alpha+\beta^{\top}\left(x-x_{j}\right)\right)>\left[V(\alpha)+e^{\alpha} w(\alpha) \beta^{\top}\left(x-x_{j}\right)\right]_{+} \geq e^{\alpha} w(\alpha)\left[\beta^{\top}\left(x-x_{j}\right)\right]_{+} .
$$

Because $F_{0}$ surrounds all $x_{i}, i=1, \ldots, n$, there exists $\gamma>0$ for which

$$
\min _{i} \inf _{\omega \in \Omega} \int\left[\left(x-x_{i}\right)^{\top} \omega\right]_{+} d F_{0}(x) \geq \gamma
$$

where $\Omega=\left\{\omega \in \mathbb{R}^{d} \mid \omega^{\top} \omega=1\right\}$, as before. Applying this bound with 45 we get

$$
-N \int V\left(\alpha+\beta^{\top}\left(x-x_{j}\right) d F_{0}(x)<-N e^{\alpha} w(\alpha) \int\left[\beta^{\top}\left(x-x_{j}\right)\right]_{+} d F_{0}(x) \leq-N e^{\alpha} w(\alpha)\|\beta\| \gamma .\right.
$$

Applying (44) and (46) in (43), we get

$$
\begin{aligned}
C^{j}(\alpha, 0)-C^{j}(\alpha, \beta) & <N V(\alpha)-N e^{\alpha} w(\alpha)\|\beta\| \gamma+n w(\alpha)\|\beta\| C \\
& =N V(\alpha)-w(\alpha)\left(N e^{\alpha} \gamma-n C\right)\|\beta\| .
\end{aligned}
$$

At the minimizer $\left(\alpha_{N}^{j}, \beta_{N}\right)$, this becomes

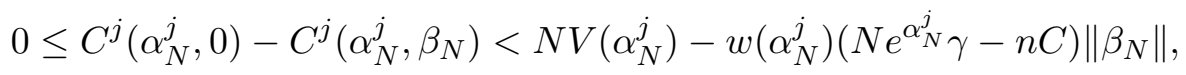

which implies

$$
\left\|\beta_{N}\right\|\left(\gamma-\frac{n C}{N} e^{-\alpha_{N}^{j}}\right) \leq \frac{V\left(\alpha_{N}^{j}\right)}{e^{\alpha_{N}^{j} w\left(\alpha_{N}^{j}\right)}} .
$$

The right side is bounded for large $\alpha_{N}^{j}$, by assumption. Through any subsequence $N_{m}$ through which $\left\|\beta_{N_{m}}\right\|$ grows without bound, this inequality is eventually violated unless $\alpha_{N_{m}}^{j} \rightarrow-\infty$. Thus, if $\left\|\beta_{N_{m}}\right\|$ is unbounded, we must have $\alpha_{N_{m}}^{j} \equiv \alpha_{N_{m}}+\beta_{N_{m}}^{\top} x_{j} \rightarrow-\infty$. Suppose $\left\|\beta_{N_{m}}\right\|$ remains bounded. We know from 41 that $\min _{i}\left\{\alpha_{N_{m}}+\beta_{N_{m}}^{\top} x_{i}\right\} \rightarrow-\infty$, so we must have $\alpha_{N_{m}} \rightarrow-\infty$, and thus we again have $\alpha_{N_{m}}+\beta_{N_{m}}^{\top} x_{j} \rightarrow-\infty$. We conclude that $\alpha_{N}+\beta_{N}^{\top} x_{j} \rightarrow-\infty$, for all $j=1, \ldots, n$, and thus $\alpha_{N}+\beta_{N}^{\top} \bar{x} \rightarrow-\infty$.

We complete the proof of Lemma 4.1 by showing that $\left\|\beta_{N}\right\|$ remains bounded. In light of Lemma B.1. boundedness of $\left\|\beta_{N}\right\|$ implies that $\alpha_{N} \rightarrow-\infty$, as required for (10). 
Lemma B.2 (Step 2). $\lim _{N \rightarrow \infty}\left\|\beta_{N}\right\|<\infty$

Proof. We will work with the centered loss, centered around $\bar{x}$,

$$
\tilde{C}(\alpha, \beta)=\sum_{i=1}^{n}-U\left(\alpha+\beta^{\top}\left(x_{i}-\bar{x}\right)\right)+N \int V\left(\alpha+\beta^{\top}(x-\bar{x})\right) d F_{0}(x),
$$

which is minimized at $\left(\tilde{\alpha}_{N}, \tilde{\beta}_{N}\right)$, with

$$
\tilde{\alpha}_{N}=\alpha_{N}+\beta_{N}^{\top} \bar{x}, \quad \tilde{\beta}_{N}=\beta_{N} .
$$

For any $\alpha \in \mathbb{R}$ and $\beta \in \mathbb{R}^{d}$,

$$
\begin{aligned}
& \tilde{C}(\alpha, 0)-\tilde{C}(\alpha, \beta) \\
& =\sum_{i=1}^{n}\left[-U(\alpha)+U\left(\alpha+\beta^{\top}\left(x_{i}-\bar{x}\right)\right)\right]+N V(\alpha)-N \int V\left(\alpha+\beta^{\top}(x-\bar{x})\right) d F_{0}(x) .
\end{aligned}
$$

We will bound the expression on the right.

Using the concavity of $U$, as in (44), we get

$$
\sum_{i=1}^{n}\left[-U(\alpha)+U\left(\alpha+\beta^{\top}\left(x_{i}-\bar{x}\right)\right)\right] \leq \sum_{i=1}^{n} w(\alpha) \beta^{\top}\left(x_{i}-\bar{x}\right)=0 ;
$$

and using the strict convexity of $V$, as in 45 , we get

$$
V\left(\alpha+\beta^{\top}(x-\bar{x})\right)>e^{\alpha} w(\alpha)\left[\beta^{\top}(x-\bar{x})\right]_{+} .
$$

As $F_{0}$ surrounds $\bar{x}$, we may pick a $\gamma>0$ such that

$$
\inf _{\omega \in \Omega} \int\left[(x-\bar{x})^{\top} \omega\right]_{+} d F_{0}(x) \geq \gamma>0 .
$$

Combining this with (51) as in (46), we get

$$
-N \int V\left(\alpha+\beta^{\top}(x-\bar{x})\right) d F_{0}(x)<-N e^{\alpha} w(\alpha)\|\beta\| \gamma .
$$

Applying (50) and (52) in (49), we get

$$
\tilde{C}(\alpha, 0)-\tilde{C}(\alpha, \beta)<N V(\alpha)-N e^{\alpha} w(\alpha)\|\beta\| \gamma
$$

Asymptotically exponential or bounded $w$. For any $\epsilon>0$, there is a $u_{0} \in \mathbb{R}$ such that

$$
(1-\epsilon) C e^{-\lambda u} \leq w(u) \leq(1+\epsilon) C e^{-\lambda u}
$$

for all $u \leq u_{0}$. Taking $\lambda=0$ covers the case of bounded $w$. The upper bound on $w(u)$ yields

$$
V(u) \leq \int_{-\infty}^{u} e^{s}(1+\epsilon) C e^{-\lambda s} d s=C \frac{1+\epsilon}{1-\lambda} e^{(1-\lambda) u}
$$


for $u \leq u_{0}$. From Lemma B.1, we have $\tilde{\alpha}_{N} \rightarrow-\infty$, so we may take $N$ large enough that $\tilde{\alpha}_{N} \leq u_{0}$. Applying (54) in (53), at the minimizer $\left(\tilde{\alpha}_{N}, \beta_{N}\right)$, we get

$$
\begin{aligned}
0 \leq \tilde{C}\left(\tilde{\alpha}_{N}, 0\right)-\tilde{C}\left(\tilde{\alpha}_{N}, \beta_{N}\right) & <N C \frac{1+\epsilon}{1-\lambda} e^{(1-\lambda) \tilde{\alpha}_{N}}-N e^{\tilde{\alpha}_{N}} w\left(\tilde{\alpha}_{N}\right)\left\|\beta_{N}\right\| \gamma \\
& \leq N C \frac{1+\epsilon}{1-\lambda} e^{(1-\lambda) \tilde{\alpha}_{N}}-N C(1-\epsilon) e^{(1-\lambda) \tilde{\alpha}_{N}}\left\|\beta_{N}\right\| \gamma \\
& =N C e^{(1-\lambda) \tilde{\alpha}_{N}}\left(\frac{1+\epsilon}{1-\lambda}-(1-\epsilon)\left\|\beta_{N}\right\| \gamma\right) .
\end{aligned}
$$

Thus, $\left\|\beta_{N}\right\| \leq \frac{1+\epsilon}{1-\epsilon} \frac{1}{\gamma(1-\lambda)}$, for all sufficiently large $N$. As $\epsilon>0$ is arbitrary, we conclude that $\limsup _{N \rightarrow \infty}\left\|\beta_{N}\right\| \leq \frac{1}{\gamma(1-\lambda)}$.

Asymptotically polynomial $w$. For any $\epsilon>0$ there is a $u_{0} \in \mathbb{R}$ such that

$$
(1-\epsilon) C|u|^{k} \leq w(u) \leq(1+\epsilon) C|u|^{k}
$$

for all $u \leq u_{0}$. The upper bound yields, for $u \leq u_{0}$,

$$
V(u) \leq(1+\epsilon) C \int_{-\infty}^{u} e^{s}|s|^{k} d s=(1+\epsilon) C\left(e^{u}|u|^{k}-k \int_{-\infty}^{u} e^{s}|s|^{k-1} d s\right) \leq(1+\epsilon) C e^{u}|u|^{k} .
$$

From Lemma B.1, we have $\tilde{\alpha}_{N} \rightarrow-\infty$, so we may take $N$ large enough that $\tilde{\alpha}_{N} \leq u_{0}$, and then (53) yields

$$
\begin{aligned}
0 \leq \tilde{C}\left(\tilde{\alpha}_{N}, 0\right)-\tilde{C}\left(\tilde{\alpha}_{N}, \beta_{N}\right) & <N V\left(\tilde{\alpha}_{N}\right)-N e^{\tilde{\alpha}_{N}} w\left(\tilde{\alpha}_{N}\right)\left\|\beta_{N}\right\| \gamma \\
& \leq N C(1+\epsilon) e^{\tilde{\alpha}_{N}}\left|\tilde{\alpha}_{N}\right|^{k}-N C(1-\epsilon) e^{\tilde{\alpha}_{N}}\left|\tilde{\alpha}_{N}\right|^{k}\left\|\beta_{N}\right\| \gamma \\
& =N C e^{\tilde{\alpha}_{N}}\left|\tilde{\alpha}_{N}\right|^{k}\left(1+\epsilon-(1-\epsilon)\left\|\beta_{N}\right\| \gamma\right) .
\end{aligned}
$$

Thus, $\left\|\beta_{N}\right\| \leq \frac{1}{\gamma} \frac{1+\epsilon}{1-\epsilon}$, for all sufficiently large $N$. As $\epsilon>0$ is arbitrary, $\lim \sup _{N}\left\|\beta_{N}\right\| \leq 1 / \gamma$.

\section{B.2 Proof of Theorem 4.2}

Proof of Theorem 4.2. Taking partial derivative of $\bar{C}$ with respect to $\alpha$ and $\beta$ using Lemma A.1. we get

$$
\begin{gathered}
\frac{\partial \bar{C}}{\partial \alpha}=\sum_{i=1}^{n}-w\left(\alpha+\beta^{\top} x_{i}\right)+N \int e^{\alpha+\beta^{\top} x} w\left(\alpha+\beta^{\top} x\right) d F_{0}(x) \\
\frac{\partial \bar{C}}{\partial \beta}=\sum_{i=1}^{n}-w\left(\alpha+\beta^{\top} x_{i}\right) x_{i}+N \int e^{\alpha+\beta^{\top} x} w\left(\alpha+\beta^{\top} x\right) x d F_{0}(x) .
\end{gathered}
$$

At the minimizer $\left(\alpha_{N}, \beta_{N}\right)$, these derivatives equal zero, so

$$
\sum_{i=1}^{n} w\left(\alpha_{N}+\beta_{N}^{\top} x_{i}\right)=N \int e^{\alpha_{N}+\beta_{N}^{\top} x} w\left(\alpha_{N}+\beta_{N}^{\top} x\right) d F_{0}(x)
$$

and

$$
\sum_{i=1}^{n} w\left(\alpha_{N}+\beta_{N}^{\top} x_{i}\right) x_{i}=N \int e^{\alpha_{N}+\beta_{N}^{\top} x} w\left(\alpha_{N}+\beta_{N}^{\top} x\right) x d F_{0}(x) .
$$


Lemma B.3. Under the conditions of Theorem 4.2.

$$
\frac{\int e^{\beta_{N}^{\top} x} x d F_{0}(x)}{\int e^{\beta_{N}^{\top} x} d F_{0}(x)} \rightarrow \bar{x} .
$$

Proof of Lemma B.3. In the asymptotically polynomial case, $w(u) \sim C|u|^{k}$, as $u \rightarrow-\infty$, for some $k>0$ and $C>0$. Taking $k=0$ covers the bounded case: $w$ is decreasing, so it approaches its maximum as $u \rightarrow-\infty$. Because $\alpha_{N} \rightarrow-\infty$ and $\beta_{N}$ is bounded, for every $x \in \mathbb{R}^{d}$ we have

$$
\frac{w\left(\alpha_{N}+\beta_{N}^{\top} x\right)}{w\left(\alpha_{N}\right)} \rightarrow 1
$$

and therefore

$$
\frac{\sum_{i} w\left(\alpha_{N}+\beta_{N}^{\top} x_{i}\right) x_{i}}{\sum_{i} w\left(\alpha_{N}+\beta_{N}^{\top} x_{i}\right)}=\frac{\sum_{i} w\left(\alpha_{N}+\beta_{N}^{\top} x_{i}\right) x_{i} / w\left(\alpha_{N}\right)}{\sum_{i} w\left(\alpha_{N}+\beta_{N}^{\top} x_{i}\right) / w\left(\alpha_{N}\right)} \rightarrow \frac{\sum_{i} x_{i}}{\sum_{i} 1}=\bar{x} .
$$

Taking the ratios of the two sides in (56)-(57), we get

$$
\frac{\int e^{\beta_{N}^{\top} x} w\left(\alpha_{N}+\beta_{N}^{\top} x\right) x d F_{0}(x)}{\int e^{\beta_{N}^{\top} x} w\left(\alpha_{N}+\beta_{N}^{\top} x\right) d F_{0}(x)} \rightarrow \bar{x} .
$$

It remains to show that the same limit holds with $w\left(\alpha_{N}+\beta_{N}^{\top} x\right)$ removed from both integrals.

Because (by Lemma B.2 $\beta_{N}$ is contained in a compact set, any subsequence of $\beta_{N}$ contains a further subsequence that converges to some $\beta$ in the compact set. We will evaluate 60 through any subsequence through which $\beta_{N} \rightarrow \beta$, dividing the numerator and denomonator by $w\left(\alpha_{N}\right)$. To bring the limit inside the integrals, we bound the integrands.

For any $\epsilon>0$, there is a $u_{0} \in \mathbb{R}$ such that (55) holds for all $u \leq u_{0}$. Take $N$ sufficiently large that $\alpha_{N} \leq u_{0}$. If $\alpha_{N}+\beta_{N}^{\top} x \leq u_{0}$, then, for large $N$,

$$
\frac{w\left(\alpha_{N}+\beta_{N}^{\top} x\right)}{w\left(\alpha_{N}\right)} \leq \frac{(1+\epsilon)}{(1-\epsilon)} \frac{\left|\alpha_{N}+\beta_{N}^{\top} x\right|^{k}}{\left|\alpha_{N}\right|^{k}} \leq \frac{(1+\epsilon)}{(1-\epsilon)}\left(1+\epsilon\|x\|^{k}\right) .
$$

If $\alpha_{N}+\beta_{N}^{\top} x>u_{0}$, then $w\left(\alpha_{N}+\beta_{N}^{\top} x\right) / w\left(\alpha_{N}\right) \leq 1$, because $w$ is decreasing, so 61 remains valid. We therefore have

$$
e^{\beta_{N}^{\top} x} \frac{w\left(\alpha_{N}+\beta_{N}^{\top} x\right)}{w\left(\alpha_{N}\right)} \leq e^{r\|x\|} \frac{(1+\epsilon)}{(1-\epsilon)}\left(1+\epsilon\|x\|^{k}\right)
$$

with $r \geq\left\|\beta_{N}\right\|$. (We saw in the proof of Lemma B.2 that we can take $r=1 / \gamma$.) By the tail condition (11), this upper bound is integrable with respect to $F_{0}$. We may therefore apply the dominated convergence theorem, recalling $(59)$, to get

$$
\int e^{\beta_{N}^{\top} x} \frac{w\left(\alpha_{N}+\beta_{N}^{\top} x\right)}{w\left(\alpha_{N}\right)} d F_{0}(x) \rightarrow \int e^{\beta^{\top} x} d F_{0}(x)
$$


whenever $\beta_{N} \rightarrow \beta$. Similarly,

$$
\int e^{\beta_{N}^{\top} x} x \frac{w\left(\alpha_{N}+\beta_{N}^{\top} x\right)}{w\left(\alpha_{N}\right)} d F_{0}(x) \rightarrow \int e^{\beta^{\top} x} x d F_{0}(x) .
$$

The same argument shows that we may interchange limit and integral in the numerator and denominator on the left side of (58). We have thus shown that whenever $\beta_{N} \rightarrow \beta$,

$$
\lim _{N \rightarrow \infty} \frac{\int e^{\beta_{N}^{\top} x} x d F_{0}(x)}{\int e^{\beta_{N}^{\top} x} d F_{0}(x)}=\frac{\int e^{\beta^{\top} x} x d F_{0}(x)}{\int e^{\beta^{\top} x} d F_{0}(x)}=\lim _{N \rightarrow \infty} \frac{\int e^{\beta_{N}^{\top} x} w\left(\alpha_{N}+\beta_{N}^{\top} x\right) x / w\left(\alpha_{N}\right) d F_{0}(x)}{\int e^{\beta_{N}^{\top} x} w\left(\alpha_{N}+\beta_{N}^{\top} x\right) / w\left(\alpha_{N}\right) d F_{0}(x)} .
$$

We know from (60) that this limit is $\bar{x}$. As this is the same for all limit points of $\beta_{N}$, the limit in (58) holds through the original sequence $\beta_{N}$.

Returning to the proof of Theorem 4.2 it remains to show the convergence of $\beta_{N}$. Because $\beta_{N}$ is contained within a compact set, any subsequence contains a further subsequence that converges. Any such limit point must be a root of $(12)$, so a root exists. To conclude the proof, we need to show that there cannot be more than one root.

Define $\psi(\beta)=\log \mathbb{E}_{0}\left[e^{\beta^{\top} X}\right]$, the cumulant generating function of $F_{0}$. Equation 12 reads $\nabla \psi\left(\beta_{*}\right)=\bar{x}$. The surrounding condition on $F_{0}$ ensures that the support of $F_{0}$ has full dimension. By Theorem 1.13(iv) of Brown [1], this implies that $\psi$ is strictly convex. Strict convexity implies that for $\beta \neq \beta_{*}$,

$$
\nabla \psi(\beta) \cdot\left(\beta_{*}-\beta\right)<\psi\left(\beta_{*}\right)-\psi(\beta)<\nabla \psi\left(\beta_{*}\right) \cdot\left(\beta_{*}-\beta\right)
$$

and thus $\nabla \psi(\beta) \neq \nabla \psi\left(\beta_{*}\right)$.

\section{B.3 Boundedness of $V(u) / e^{u} w(u)$}

Definition 2 requires an upper bound on $V(u) / e^{u} w(u)$, and this condition is used in (47). The following lemma shows that this condition is satisfied by a broad family of weight functions, including $w(u)=C e^{-\lambda_{1} u}, \lambda_{1} \in(0,1)$, and $w(u)=C u^{-k}, k>0$, for large $u$.

Lemma B.4. Suppose there is an increasing log-convex function $g$ for which $C g(u) \leq e^{u} w(u) \leq$ $g(u)$, for all $u \geq u_{0}$, for some $u_{0} \in \mathbb{R}$ with $g^{\prime}\left(u_{0}\right) \neq 0$, and some $C>0$. Then $V(u) / e^{u} w(u)$ is bounded above on $\left[u_{0}, \infty\right)$.

Proof. Theorem 2.1 of Gill, Pearce, and Pečarić [10] provides an upper bound on the integral of a log-convex function $g$, which yields, for any $u>u_{0}$,

$$
V(u)=V\left(u_{0}\right)+\int_{u_{0}}^{u} e^{s} w(s) d s \leq V\left(u_{0}\right)+\int_{u_{0}}^{u} g(s) d s \leq V\left(u_{0}\right)+\frac{\left(u-u_{0}\right)\left(g(u)-g\left(u_{0}\right)\right)}{\log g(u)-\log g\left(u_{0}\right)} .
$$


Since $g\left(u_{0}\right) \geq e^{u_{0}} w\left(u_{0}\right) \geq 0$, the bound remains valid if we remove $g\left(u_{0}\right)$ from the numerator

of the last term. Convexity of $\log g$ implies that $\log g(u)-\log g\left(u_{0}\right) \geq\left(\log g\left(u_{0}\right)\right)^{\prime}\left(u-u_{0}\right)$, and $\left(\log g\left(u_{0}\right)\right)^{\prime}=g^{\prime}\left(u_{0}\right) / g\left(u_{0}\right)$, so

$$
\frac{V(u)}{e^{u} w(u)} \leq \frac{V\left(u_{0}\right)}{e^{u} w(u)}+\frac{g(u)}{e^{u} w(u)} \frac{\left(u-u_{0}\right)}{\log g(u)-\log g\left(u_{0}\right)} \leq \frac{V\left(u_{0}\right)}{e^{u_{0}} w\left(u_{0}\right)}+\frac{1}{C} \frac{g\left(u_{0}\right)}{g^{\prime}\left(u_{0}\right)} .
$$

\section{B.4 Proof of Theorem 4.3}

Proof of Theorem 4.3. We can rewrite the first-order conditions (56)-(57) as

$$
\frac{\sum_{i=1}^{n} w\left(\alpha_{N}+\beta_{N}^{\top} x_{i}\right) x_{i}}{\sum_{i=1}^{n} w\left(\alpha_{N}+\beta_{N}^{\top} x_{i}\right)}=\frac{\int e^{\beta_{N}^{\top} x} w\left(\alpha_{N}+\beta_{N}^{\top} x\right) x d F_{0}(x)}{\int e^{\beta_{N}^{\top} x} w\left(\alpha_{N}+\beta_{N}^{\top} x\right) d F_{0}(x)} .
$$

We will take limits on the two sides of this equation.

Since $\alpha_{N}+\beta_{N}^{\top} \bar{x} \rightarrow-\infty$ and $\lim \sup _{N}\left\|\beta_{N}\right\|<\infty, \alpha_{N}+\beta_{N}^{\top} x \rightarrow-\infty$ for any fixed $x$. Because $w(u) \sim C e^{-\lambda u}$, as $u \rightarrow-\infty$, we have, for every $x \in \mathbb{R}^{d}$,

$$
\frac{w\left(\alpha_{N}+\beta_{N}^{\top} x\right)}{w\left(\alpha_{N}\right)} \rightarrow e^{-\lambda \beta^{\top} x},
$$

for any subsequence through which $\beta_{N} \rightarrow \beta$. We then also have

$$
\frac{\sum_{i=1}^{n} w\left(\alpha_{N}+\beta_{N}^{\top} x_{i}\right) x_{i}}{\sum_{i=1}^{n} w\left(\alpha_{N}+\beta_{N}^{\top} x_{i}\right)} \rightarrow \frac{\sum_{i=1}^{n} e^{-\lambda \beta^{\top} x_{i}} x_{i}}{\sum_{i=1}^{n} e^{-\lambda \beta^{\top} x_{i}}}
$$

through any such subsequence.

Turning to the right side of (64), for any $\epsilon>0$ and all sufficiently large $N$, we have

$$
\frac{w\left(\alpha_{N}+\beta_{N}^{\top} x\right)}{w\left(\alpha_{N}\right)} \leq \max \left\{1,(1+\epsilon) e^{-\lambda \beta_{N}^{\top} x}\right\}
$$

for all $x \in \mathbb{R}^{d}$, arguing as we did in 61. It follows that

$$
e^{\beta_{N}^{\top} x} \frac{w\left(\alpha_{N}+\beta_{N}^{\top} x\right)}{w\left(\alpha_{N}\right)} \leq \max \left\{e^{\beta_{N}^{\top} x},(1+\epsilon) e^{(1-\lambda) \beta_{N}^{\top} x}\right\} \leq(1+\epsilon) e^{r\|x\|},
$$

with $r \geq\left\|\beta_{N}\right\|$. (We saw in the proof of Lemma B.2 that we may take $r=1 / \gamma(1-\lambda)$ ) By the tail condition (11), this upper bound is integrable with respect to $F_{0}$. The same is true if we multiply both sides by $\|x\|$. We may therefore apply the dominated convergence theorem and 65) to conclude that through any subsequence with $\beta_{N} \rightarrow \beta$,

$$
\frac{\int e^{\beta_{N}^{\top} x} w\left(\alpha_{N}+\beta_{N}^{\top} x\right) x d F_{0}(x)}{\int e^{\beta_{N}^{\top} x} w\left(\alpha_{N}+\beta_{N}^{\top} x\right) d F_{0}(x)} \rightarrow \frac{\int e^{(1-\lambda) \beta^{\top} x} x d F_{0}(x)}{\int e^{(1-\lambda) \beta^{\top} x} d F_{0}(x)} .
$$


Applying (66) and (67) to the two sides of $(64)$, we conclude that any limit point of $\beta_{N}$ must be a solution to $(13)$.

Because $\beta_{N}$ is contained in a compact set, it has at least one limit point, so (13) has at least one solution. We need to show that it cannot have more than one solution. Define the cumulant generating function $\psi(\beta)=\log \mathbb{E}\left[e^{\beta^{\top} W}\right]$ of $W=(1-\lambda) X_{0}-\lambda X_{1}$, where $X_{0} \sim F_{0}$, $X_{1}$ is uniform over of $x_{1}, \ldots, x_{n}$, and $X_{0}, X_{1}$ are independent. Then (13) reads $\nabla \psi\left(\beta_{*}\right)=0$. Uniqueness follows by the argument used at the end of the proof of Theorem 4.2 ,

\section{B.5 Proof of Theorem 4.4}

Proof. With $w$ as in (14), the first-order conditions (56)-(57) become

$$
\sum_{i=1}^{n}-e^{-\lambda\left(\alpha_{N}+\beta_{N}^{\top} x_{i}\right)}+N \int e^{(1-\lambda)\left(\alpha_{N}+\beta_{N}^{\top} x\right)} d F_{0}(x)=0
$$

and

$$
\sum_{i=1}^{n}-e^{-\lambda\left(\alpha_{N}+\beta_{N}^{\top} x_{i}\right)} x_{i}+N \int x e^{(1-\lambda)\left(\alpha_{N}+\beta_{N}^{\top} x\right)} d F_{0}(x)=0 .
$$

Grouping together terms with $\alpha_{N}$, we get

$$
N e^{\alpha_{N}}=\frac{\sum_{i=1}^{n} e^{-\lambda \beta_{N}^{\top} x_{i}}}{\int e^{(1-\lambda) \beta_{N}^{\top} x} d F_{0}(x)}=\frac{\sum_{i=1}^{n} x_{i} e^{-\lambda \beta_{N}^{\top} x_{i}}}{\int x e^{(1-\lambda) \beta_{N}^{\top} x} d F_{0}(x)} .
$$

The second equality shows that $\beta_{N}$ satisfies 13 for all $N$, and we know from Theorem 4.3 that (13) has just one solution, $\beta_{*}$. With $\beta_{N} \equiv \beta_{*}$, the first equality shows that $\alpha_{N}$ is a constant minus $\log N$.

\section{Supplementary Tables}

\section{C.1 Freddie Mac Dataset AUC}

Tables 36 report AUC values for training, validation, and test sets for four choices of classifiers. Results are indexed by training year, so test results for 2003, for example, are based on defaults predicted for the first quarter of 2006. In all cases, the test and validation AUCs are close to the training AUCs and above 0.8.

\section{C.2 Freddie Mac Testing TPR}

Table 7 shows true positive rates in test data for four classifiers. In each case, the classification threshold was set in the training data to achieve a TPR of $99 \%$. The results show that the same thresholds achieve very similar TPRs in the test data. Results are indexed by training year, so test results for 2003, for example, are based on defaults predicted for the first quarter of 2006 . 


\begin{tabular}{l|rrr}
\hline Year & Train & Val & Test \\
\hline 2003 & 0.8917 & 0.8684 & 0.8868 \\
2004 & 0.8478 & 0.8562 & 0.8495 \\
2005 & 0.8446 & 0.8450 & 0.8447 \\
2006 & 0.8298 & 0.8276 & 0.8293 \\
2007 & 0.8263 & 0.8223 & 0.8255 \\
2008 & 0.8486 & 0.8516 & 0.8492 \\
2009 & 0.8759 & 0.8743 & 0.8756 \\
2010 & 0.8454 & 0.8514 & 0.8467 \\
2011 & 0.8666 & 0.8721 & 0.8677 \\
2012 & 0.8751 & 0.8631 & 0.8731 \\
2013 & 0.8524 & 0.8611 & 0.8542 \\
\hline
\end{tabular}

Table 3: AUC, Logistic Regression

\begin{tabular}{r|rrr}
\hline Year & Train & Val & Test \\
\hline 2003 & 0.8929 & 0.8700 & 0.8881 \\
2004 & 0.8515 & 0.8554 & 0.8523 \\
2005 & 0.8533 & 0.8546 & 0.8535 \\
2006 & 0.8326 & 0.8302 & 0.8321 \\
2007 & 0.8286 & 0.8247 & 0.8278 \\
2008 & 0.8511 & 0.8541 & 0.8517 \\
2009 & 0.8797 & 0.8782 & 0.8794 \\
2010 & 0.8479 & 0.8502 & 0.8484 \\
2011 & 0.8703 & 0.8782 & 0.8719 \\
2012 & 0.8755 & 0.8648 & 0.8737 \\
2013 & 0.8534 & 0.8629 & 0.8555 \\
\hline
\end{tabular}

Table 5: AUC, $\lambda=0.5$

\begin{tabular}{l|rrr}
\hline Year & Train & Val & Test \\
\hline 2003 & 0.8926 & 0.8706 & 0.8881 \\
2004 & 0.8491 & 0.8558 & 0.8505 \\
2005 & 0.8479 & 0.8493 & 0.8482 \\
2006 & 0.8305 & 0.8278 & 0.8300 \\
2007 & 0.8282 & 0.8245 & 0.8275 \\
2008 & 0.8502 & 0.8531 & 0.8508 \\
2009 & 0.8788 & 0.8770 & 0.8785 \\
2010 & 0.8473 & 0.8506 & 0.8480 \\
2011 & 0.8703 & 0.8761 & 0.8714 \\
2012 & 0.8755 & 0.8639 & 0.8736 \\
2013 & 0.8532 & 0.8620 & 0.8551 \\
\hline
\end{tabular}

Table 4: AUC, $\lambda=0.1$

\begin{tabular}{l|rrr}
\hline Year & Train & Val & Test \\
\hline 2003 & 0.8892 & 0.8656 & 0.8843 \\
2004 & 0.8503 & 0.8536 & 0.8510 \\
2005 & 0.8528 & 0.8541 & 0.8530 \\
2006 & 0.8308 & 0.8288 & 0.8304 \\
2007 & 0.8263 & 0.8222 & 0.8255 \\
2008 & 0.8495 & 0.8526 & 0.8501 \\
2009 & 0.8773 & 0.8758 & 0.8770 \\
2010 & 0.8441 & 0.8452 & 0.8443 \\
2011 & 0.8570 & 0.8631 & 0.8582 \\
2012 & 0.8662 & 0.8574 & 0.8647 \\
2013 & 0.8503 & 0.8596 & 0.8523 \\
\hline
\end{tabular}

Table 6: AUC, $\lambda=0.9$

\section{Delta Function Weight}

The loss function defined by setting, for some $u_{0} \in \mathbb{R}$,

$$
U(s)=-\mathbf{1}\left\{s \leq u_{0}\right\}, \quad V(s)=e^{u_{0}} \mathbf{1}\left\{s>u_{0}\right\},
$$

can be interpreted as taking $w$ to be a delta function with unit mass at $u_{0}$. This case leads to the objective in (15) and its counterpart

$$
\bar{C}(\alpha, \beta)=\sum_{i=1}^{n} \mathbf{1}\left\{\alpha+\beta^{\top} x_{i} \leq u_{0}\right\}+N e^{u_{0}} \mathbb{P}_{0}\left(\alpha+\beta^{\top} X>u_{0}\right) .
$$

As discussed in Example 2 in Section 2.4 of Eguchi and Copas [5], through approrpriate choice of $u_{0}, C(\alpha, \beta)$ can be interpreted as balancing misclassification costs. However, $\bar{C}(\alpha, \beta)$ is not convex, and we will show that the resulting linear discriminant function degenerates under imbalance, in the sense that $\beta_{N}=0$ for all sufficiently large $N$. 


\begin{tabular}{rrrrr}
\hline Year & Logistic & $\lambda=0.1$ & $\lambda=0.5$ & $\lambda=0.9$ \\
\hline 2003 & 98.83 & 98.83 & 98.83 & 98.76 \\
2004 & 98.86 & 98.93 & 98.86 & 98.93 \\
2005 & 99.12 & 99.12 & 99.07 & 99.02 \\
2006 & 99.02 & 98.98 & 98.98 & 98.93 \\
2007 & 99.02 & 99.00 & 99.02 & 99.01 \\
2008 & 98.98 & 98.98 & 98.98 & 99.01 \\
2009 & 99.09 & 99.04 & 98.95 & 99.00 \\
2010 & 99.10 & 99.10 & 99.10 & 98.97 \\
2011 & 99.34 & 98.90 & 98.90 & 98.90 \\
2012 & 98.70 & 98.70 & 98.70 & 98.44 \\
2013 & 99.21 & 99.21 & 99.21 & 99.01 \\
\hline
\end{tabular}

Table 7: TPR (in percent) in test data using classification thresholds that achieve $\mathrm{TPR}=99 \%$ in training data.

For any $\alpha, \beta$, we have $\bar{C}(\alpha, \beta) \geq 0$, and for any $\alpha \leq u_{0}$, we have $\bar{C}(\alpha, 0)=n$. In particular, then, if $\left(\alpha_{N}, \beta_{N}\right)$ minimizes $\bar{C}$,

$$
0 \leq \bar{C}\left(\alpha_{N}, \beta_{N}\right) \leq \bar{C}(\alpha, 0)=n
$$

for all $\alpha \leq u_{0}$.

Lemma D.1. Suppose $F_{0}$ surrounds $x_{j}$ for some $1 \leq j \leq n$, and let $\delta>0$ be such that, for $\Omega=\left\{\omega \in \mathbb{R}^{d} \mid \omega^{\prime} \omega=1\right\}$,

$$
\inf _{\omega \in \Omega} \int_{\left(x-x_{j}\right)^{\top} \omega \geq 0} d F_{0}(x) \geq \delta
$$

Then $\alpha_{N}+\beta_{N}^{\top} x_{j} \leq u_{0}$, for all $N>n / e^{u_{0}} \delta$.

Proof. At any $(\alpha, \beta)$ for which $\alpha+\beta^{\top} x_{j}>u_{0}$, we have

$$
\begin{aligned}
\mathbb{P}_{0}\left(\alpha+\beta^{\top} X>u_{0}\right) & =\int_{\alpha+\beta^{\top} x>u_{0}} d F_{0}(x) \\
& =\int_{\beta^{\top}\left(x-x_{j}\right)>u_{0}-\left(\alpha+\beta^{\top} x_{j}\right)} d F_{0}(x) \\
& \geq \int_{\beta^{\top}\left(x-x_{j}\right) \geq 0} d F_{0}(x) \\
& \geq \delta
\end{aligned}
$$

For $N>n / e^{u_{0}} \delta$, 68) then implies $\bar{C}(\alpha, \beta)>n$. But we know $\bar{C}\left(\alpha_{N}, \beta_{N}\right) \leq n$ from (69), so we must have $\alpha_{N}+\beta_{N}^{\top} x_{j} \leq u_{0}$, for all $N>n / e^{u_{0}} \delta$.

With this lemma, we have the following result. 
Proposition D.1. Suppose $F_{0}$ surrounds every $x_{i}, i=1,2, \ldots, n$. Then for all sufficiently large $N$ large, every pair $(\alpha, \beta)$ of the form $\alpha \leq u_{0}, \beta=0$ is a global minimizer of $\bar{C}$.

Proof. If $\left(\alpha_{N}, \beta_{N}\right)$ minimizes $\bar{C}$, then we know from the previous lemma that $\alpha_{N}+\beta_{N}^{\top} x_{i} \leq u_{0}$, for all $i=1, \ldots, n$, for all $N>n / e^{u_{0}} \delta$. It follows from 68 that $\bar{C}\left(\alpha_{N}, \beta_{N}\right) \geq n$. At any $\alpha \leq u_{0}$, (68) also shows that $\bar{C}(\alpha, 0)=n$. Thus, every $(\alpha, 0), \alpha \leq u_{0}$, is a global minimizer if $N>n / e^{u_{0}} \delta$.

We conclude from this result that the objective (68) degenerates under sufficient imbalance, in the sense that it returns $\beta_{N}=0$, for all sufficiently large $N$. The linear discriminant function $\alpha_{N}+\beta_{N}^{\top} x$ assigns the same value $\alpha_{N}$ to every observation, and $\alpha_{N}$ could be any value less than or equal to $u_{0}$. 